ANALYSIS \& PDE Volume $8 \quad$ No. $8 \quad 2015$ (1)

IOAN BEJENARU, ZiHUA GUO, SEBASTIAN HERR AND KENJI NAKANISHI WELL-POSEDNESS AND SCATTERING FOR
THE ZAKHAROV SYSTEM IN FOUR DIMENSIONS TO ZAKHAROV SYSTEM IN TOUR DIMENSIONS 


\title{
WELL-POSEDNESS AND SCATTERING FOR THE ZAKHAROV SYSTEM IN FOUR DIMENSIONS
}

\author{
IoAn Bejenaru, Zihua Guo, Sebastian Herr And Kenji NaKanishi
}

\begin{abstract}
The Cauchy problem for the Zakharov system in four dimensions is considered. Some new wellposedness results are obtained. For small initial data, global well-posedness and scattering results are proved, including the case of initial data in the energy space. None of these results are restricted to radially symmetric data.
\end{abstract}

\section{Introduction and main results}

Let $\alpha>0$. The Zakharov system

$$
\left\{\begin{array}{l}
i \dot{u}-\Delta u=n u \\
\ddot{n} / \alpha^{2}-\Delta n=-\Delta|u|^{2}
\end{array}\right.
$$

with initial data

$$
u(0, x)=u_{0}, \quad n(0, x)=n_{0}, \quad \dot{n}(0, x)=n_{1}
$$

is considered as a simplified mathematical model for Langmuir waves in a plasma, which couples the envelope $u: \mathbb{R}^{1+d} \rightarrow \mathbb{C}$ of the electric field and the ion density $n: \mathbb{R}^{1+d} \rightarrow \mathbb{R}$, neglecting magnetic effects and the vector field character of the electric field; see [Sulem and Sulem 1999, Chapter V; Zakharov 1972].

The parameter $\alpha>0$ is called the ion sound speed. Formally, as $\alpha \rightarrow \infty,(1-1)$ reduces to the focusing cubic Schrödinger equation

$$
i \dot{u}-\Delta u=|u|^{2} u,
$$

which is energy-critical in dimension $d=4$; see for example [Kenig and Merle 2006; Killip and Visan 2010; Dodson 2014] and the references therein concerning recent developments on global-well-posedness, blow-up and scattering for (1-3). For rigorous results on the subsonic limit (as $\alpha \rightarrow \infty$ ) of (1-1) to (1-3) we refer the reader to [Schochet and Weinstein 1986; Ozawa and Tsutsumi 1992; Masmoudi and Nakanishi 2008].

Strong solutions $(u, n)$ of the Zakharov system preserve the mass

$$
\int_{\mathbb{R}^{d}}|u|^{2} d x=\int_{\mathbb{R}^{d}}\left|u_{0}\right|^{2} d x
$$

MSC2010: 35L70, 35Q55.

Keywords: nonlinear wave equation, nonlinear Schrödinger equation, Zakharov system, well-posedness, scattering. 
and the energy, with $D:=\sqrt{-\Delta}$,

$$
E(u, n, \dot{n})=\int_{\mathbb{R}^{d}}|\nabla u|^{2}+\frac{\left|D^{-1} \dot{n}\right|^{2}}{2 \alpha^{2}}+\frac{|n|^{2}}{2}-n|u|^{2} d x=E\left(u_{0}, n_{0}, n_{1}\right) .
$$

In view of (1-5), a natural space for the initial data is the energy space

$$
\left(u_{0}, n_{0}, n_{1}\right) \in H^{1}\left(\mathbb{R}^{d}\right) \times L^{2}\left(\mathbb{R}^{d}\right) \times \dot{H}^{-1}\left(\mathbb{R}^{d}\right) .
$$

For initial data in the energy space, the Zakharov system is known to be globally well-posed if $d=1$ (see [Ginibre et al. 1997]) and locally well-posed if $d=2,3$ (see [Bourgain and Colliander 1996]). A low regularity local well-posedness theory has been developed in [Ginibre et al. 1997] in all dimensions, with further extensions in [Bejenaru et al. 2009] if $d=2$, and in [Bejenaru and Herr 2011] if $d=3$; see also the references therein for previous work. In the case of the torus $\mathbb{T}^{d}$, well-posedness results were proved in [Takaoka 1999; Kishimoto 2013].

In [Merle 1996] blow-up results in finite or infinite time for initial data of negative energy were proved if $d=3$ and, if $d=2$, blow-up in finite time was derived in [Glangetas and Merle 1994a; 1994b]. Concerning the final data problem in weighted Sobolev spaces, we refer to [Shimomura 2004; Ginibre and Velo 2006; Ozawa and Tsutsumi 1993/94].

Recently, the asymptotic behavior as $t \rightarrow \infty$ for the initial data problem was studied in dimension $d=3$ : In [Guo and Nakanishi 2014], small-data energy scattering in the radial case was obtained by using a normal form technique and the improved Strichartz estimates for radial functions from [Guo and Wang 2014]. In [Guo et al. 2013], a dichotomy between scattering and grow-up was obtained for radial solutions with energy below the ground state energy. In the nonradial case in dimension $d=3$, scattering was obtained in [Hani et al. 2013] under the assumption that the initial data are small enough and have sufficient regularity and decay. This result was improved recently in [Guo et al. 2014a; Guo 2014], where scattering was shown for small initial data belonging to the energy space with some additional angular regularity.

In the present paper, we continue the analysis of the initial value problem (1-1) and focus on the energy-critical dimension $d=4$. In particular, we will address the small-data global well-posedness and scattering problem in the energy space, i.e.,

$$
\left(u_{0}, n_{0}, n_{1}\right) \in H^{1}\left(\mathbb{R}^{4}\right) \times L^{2}\left(\mathbb{R}^{4}\right) \times \dot{H}^{-1}\left(\mathbb{R}^{4}\right),
$$

with no additional symmetry or decay assumption.

We reduce the wave equation to a first-order equation as usual: let

$$
N:=n-\frac{i D^{-1} \dot{n}}{\alpha}
$$

then $n=\operatorname{Re} N=\frac{1}{2}(N+\bar{N})$ and the Zakharov system for $(u, N)$ reads as follows:

$$
\left\{\begin{array}{l}
\left(i \partial_{t}-\Delta\right) u=\frac{1}{2} N u+\frac{1}{2} \bar{N} u \\
\left(i \partial_{t}+\alpha D\right) N=\alpha D|u|^{2}
\end{array}\right.
$$


The Hamiltonian then becomes

$$
E(u, n, \dot{n})=E_{Z}(u, N):=\int_{\mathbb{R}^{4}}|\nabla u|^{2}+\frac{1}{2}|N|^{2}-\operatorname{Re} N|u|^{2} d x .
$$

We will restrict ourselves to the system (1-9). Our first main result is a small-data global well-posedness and scattering result.

Theorem 1.1. There exists $\varepsilon_{0}=\varepsilon_{0}(\alpha)>0$ such that, for any $(s, l) \in \mathbb{R}^{2}$ satisfying $(s, l)=(1,0)$ or

$$
l \geq 0, \quad s<4 l+1, \quad(s, l) \neq(2,3), \quad \max \left(\frac{1}{2}(l+1), l-1\right) \leq s \leq \min \left(l+2,2 l+\frac{11}{8}\right)
$$

and any initial data $\left(u_{0}, N_{0}\right) \in H^{s}\left(\mathbb{R}^{4}\right) \times H^{l}\left(\mathbb{R}^{4}\right)$ satisfying

$$
\left\|\left(u_{0}, N_{0}\right)\right\|_{H^{1 / 2}\left(\mathbb{R}^{4}\right) \times L^{2}\left(\mathbb{R}^{4}\right)}<\varepsilon_{0},
$$

there exists a unique global solution $(u, N) \in C\left(\mathbb{R} ; H^{s}\left(\mathbb{R}^{4}\right) \times H^{l}\left(\mathbb{R}^{4}\right)\right)$ of (1-9) with some space-time integrability. The solution map is continuous in the norms

$$
H^{s} \times H^{l} \rightarrow L^{\infty}\left(\mathbb{R} ; H^{s} \times H^{l}\right), \quad\left(u_{0}, N_{0}\right) \mapsto(u, N) .
$$

Moreover, there exist $\left(u^{ \pm}, N^{ \pm}\right) \in H^{s}\left(\mathbb{R}^{4}\right) \times H^{l}\left(\mathbb{R}^{4}\right)$ such that

$$
\lim _{t \rightarrow \pm \infty}\left(\left\|u(t)-S(t) u^{ \pm}\right\|_{H^{s}}+\left\|N(t)-W_{\alpha}(t) N^{ \pm}\right\|_{H^{l}}\right)=0
$$

where $S(t)=e^{-i t \Delta}$ and $W_{\alpha}(t)=e^{i t \alpha D}$ are the free propagators.

In the above statement, we need the space-time integrability to ensure uniqueness. For example, for any $T>0$,

$$
u \in L^{2}\left((0, T) ; B_{4,2}^{1 / 2}\left(\mathbb{R}^{4}\right)\right)
$$

is sufficient for uniqueness on $[0, T]$, where $B_{4,2}^{1 / 2}$ is the inhomogeneous Besov space. See Propositions 3.1, 5.1 and 5.2 for more detail on the space-time integrability.

Very recently, we learned about independent work of Kato and Tsugawa [ $\geq 2015]$. By a different method, they prove the small data scattering for $l=s-\frac{1}{2} \geq 0$, using bilinear estimates in $U^{p}-V^{p}$ spaces for the standard iteration. While their iteration scheme is more direct, our estimates are more elementary and we cover a wider range of $(s, l)$.

Our second result is a large-data local well-posedness result for the same range of regularity $(s, l)$ as above, except for the energy space $H^{1}\left(\mathbb{R}^{4}\right) \times L^{2}\left(\mathbb{R}^{4}\right)$.

Theorem 1.2. Let $(s, l) \in \mathbb{R}^{2}$ satisfy (1-11). Then, for any $\left(u_{0}, N_{0}\right) \in H^{s}\left(\mathbb{R}^{4}\right) \times H^{l}\left(\mathbb{R}^{4}\right)$, there exists $T=T\left(u_{0}, N_{0}\right)>0$ and a unique local solution $(u, N) \in C\left([-T, T] ; H^{s}\left(\mathbb{R}^{4}\right) \times H^{l}\left(\mathbb{R}^{4}\right)\right)$ to $(1-9)$ satisfying some space-time integrability; (1-15) is enough for the uniqueness. Both $T>0$ and $(u, N)$ depend continuously on $\left(u_{0}, N_{0}\right)$.

In dimension $d=4$, Ginibre, Tsutsumi and Velo [Ginibre et al. 1997] proved local well-posedness in the range $l \leq s \leq l+1, l>0,2 s>l+1$; see Figure 1 (right). Their method is the standard Picard iteration argument in the $X^{s, b}$ spaces. Theorem 1.2 gives further local well-posedness results in a new 

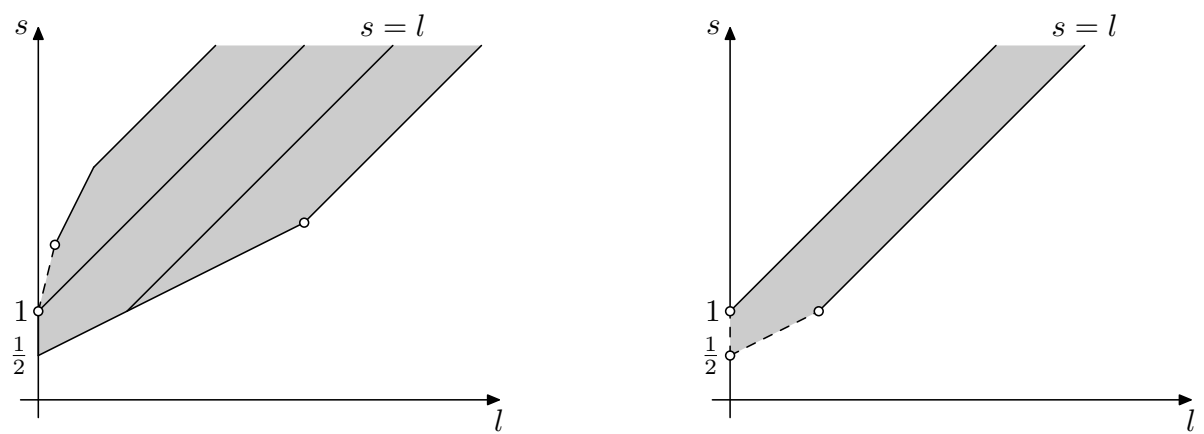

Figure 1. Left: the range of $(s, l)$ obtained in Theorems 1.1 and 1.2. Right: the range of $(s, l)$ obtained in [Ginibre et al. 1997].

region, indicated in Figure 1 (left), while Theorem 1.1 covers the same range of exponents as well as the energy space $(s, l)=(1,0)$, which is missing from the large-data result, Theorem 1.2.

The proofs for Theorems 1.1 and 1.2 use the normal form technique and Strichartz estimates as in [Guo and Nakanishi 2014] and the follow-up papers [Guo et al. 2013; 2014a; Guo 2014] and related work on the Klein-Gordon-Zakharov system [Guo et al. 2014b; 2014c]. Our argument is somewhat simpler than [Ginibre et al. 1997] and it also implies some scattering results.

There is a qualitative difference in our proof between $s<l+1$ and $s>l+1$. Since the Strichartz norm of $W_{\alpha}(t)$ is worse than that of $S(t)$, for $s<l+1$ we use only the $H_{x}^{l}$ norm for $N$, while keeping the full Strichartz norm for $u$. For $s>l+1$, however, this strategy is prevented by the normal form of $u$, so we need to modify the Strichartz norm for $u$, and to use that of $N$. Consequently, we cannot recover all the Strichartz norms of $S(t)$ for $u$, in spite of the scattering. See Proposition 5.2 for the precise statement. This is consistent with the fact that [Ginibre et al. 1997] is restricted to $s \leq l+1$ and $X^{s, b}$ implies the full range of Strichartz norm.

The energy space $(s, l)=(1,0)$ is at the intersection of $s=l+1$ and $l=0$, where our multilinear estimates actually break down. More precisely, we cannot close any Strichartz bound for the normal form of $u$ when $(s, l)=(1,0)$. This is why $(1,0)$ is excluded from Theorem 1.2. Fortunately enough, with the help of the conservation law (1-10) and using the well-posedness in nearby $(s, l)$, we are still able to show global well-posedness and scattering in the energy space $(s, l)=(1,0)$ for small data as in Theorem 1.1. Since the limit NLS (1-3) is critical in the energy space $H^{1}\left(\mathbb{R}^{4}\right)$, it may have blow-up with bounded $H^{1} \times L^{2}$ norm for large data, which suggests that there may be essential difference between large and small data.

At the other excluded endpoint, $(s, l)=(2,3)$, we can prove a strong ill-posedness result, both by instant exit and by nonexistence.

Theorem 1.3. There exists a radial function $u_{0} \in H^{2}\left(\mathbb{R}^{4}\right)$ such that, for any $\varepsilon>0$, any $N_{0} \in H^{3}\left(\mathbb{R}^{4}\right)$, and any $T_{0}>0$, the system (1-9) has no solution $(u, N) \in C\left(\left[0, T_{0}\right] ; \mathscr{Y}^{\prime}\left(\mathbb{R}^{4}\right)^{2}\right)$ satisfying $(u(0), N(0))=$ $\left(\varepsilon u_{0}, N_{0}\right)$, the equation (1-9) in the distribution sense, and

$$
(u, N) \in L^{2}\left(\left(0, T_{0}\right) ; H^{1}\left(\mathbb{R}^{4}\right) \times H^{3}\left(\mathbb{R}^{4}\right)\right) .
$$


Moreover, the unique local solution $(u, N) \in C\left([-T, T] ; H^{2} \times H^{2}\right)$ given by Theorem 1.2 satisfies $N(t) \notin H^{3}\left(\mathbb{R}^{4}\right)$ for all $t \in[-T, T] \backslash\{0\}$.

Note that (1-16) is weaker than the usual weak solutions, as it does not require $(u(t), N(t)) \in H^{s} \times H^{l}$ for all $t$ near 0 . The above ill-posedness is due to the mismatch of regularity between $u$ and $N$ in the normal form for $N$.

The rest of paper is organized as follows. In Section 2, we recall the normal form reduction from [Guo and Nakanishi 2014], and then gather multilinear estimates used in the later sections. They easily follow from the Littlewood-Paley decomposition, Coifman-Meyer bilinear estimate, Strichartz and Sobolev inequalities. Using these estimates and the standard contraction argument, we first prove the small data scattering in $H^{s} \times H^{l}$ for $s \leq l+1$ in Section 3, and then the local well-posedness for large data in $H^{1 / 2} \times L^{2}$ in Section 4. In Section 5, we extend these results to higher regularity by persistence of regularity, except for the energy space $(s, l)=(1,0)$. Theorem 1.1 for $(s, l) \neq(1,0)$ follows from Propositions 3.1, 5.1 and 5.2. Similarly, Theorem 1.2 follows from Propositions 4.2, 5.1 and 5.2. In Section 6 , we prove Theorem 1.1 in the energy space $(s, l)=(1,0)$, using the results in $(s, 0)$ for $s<1$ and in $(1, l)$ for $l>0$. In Section 7, we prove the ill-posedness result, Theorem 1.3 at $(s, l)=(2,3)$.

\section{Normal form and multilinear estimates}

In this section, we set up integral equations and basic estimates for solving the equation. Our analysis is based on the normal form reduction devised in [Guo and Nakanishi 2014].

2A. Review of the normal form reduction and notation from [Guo and Nakanishi 2014]. Let $\hat{\phi}=\mathscr{F} \phi$ denote the Fourier transform of $\phi$. We use $S(t)$ and $W_{\alpha}(t)$ to denote the Schrödinger and wave semigroup, respectively:

$$
S(t) \phi=\mathscr{F}^{-1}\left(e^{i t|\xi|^{2}} \hat{\phi}\right) \quad \text { and } \quad W_{\alpha}(t) \phi=\mathscr{F}^{-1}\left(e^{i \alpha t|\xi|} \hat{\phi}\right)
$$

Fix a radial, smooth, bump function $\eta_{0}: \mathbb{R}^{4} \rightarrow[0,1]$ with support in the ball $B_{\frac{8}{5}}(0)$, which is equal to 1 in the smaller ball $B_{4 / 5}(0)$. For $k \in \mathbb{Z}$, let $\chi_{k}(\xi)=\eta_{0}\left(\xi / 2^{k}\right)-\eta_{0}\left(\xi / 2^{k-1}\right)$ and $\chi_{\leq k}(\xi)=\eta_{0}\left(\xi / 2^{k}\right)$, and let $P_{k}$ and $P_{\leq k}$ denote the corresponding Fourier multipliers.

For two functions $u$ and $v$, and a fixed $K \in \mathbb{N}, K \geq 5$, we define the paraproduct-type operators

$$
(u v)_{L H}:=\sum_{k \in \mathbb{Z}}\left(P_{\leq k-K} u\right)\left(P_{k} v\right), \quad(u v)_{H L}:=(v u)_{L H}, \quad(u v)_{H H}:=\sum_{\substack{k_{1}-k_{2} \mid \leq K-1 \\ k_{1}, k_{2} \in \mathbb{Z}}}\left(P_{k_{1}} u\right)\left(P_{k_{2}} v\right),
$$

so that $u v=(u v)_{L H}+(u v)_{H L}+(u v)_{H H}$. We also define

$$
\begin{aligned}
& (u v)_{\alpha L}:=\sum_{\substack{k-\log _{2} \alpha \mid \leq 1, k \in \mathbb{Z}}}\left(P_{k} u\right)\left(P_{\leq k-K} v\right), \quad(u v)_{L \alpha}:=(v u)_{\alpha L}, \\
& (u v)_{X L}:=\sum_{\substack{\left|k-\log _{2} \alpha\right|>1, k \in \mathbb{Z}}}\left(P_{k} u\right)\left(P_{\leq k-K} v\right), \quad(u v)_{L X}:=(v u)_{X L},
\end{aligned}
$$

so that $(u v)_{H L}=(u v)_{\alpha L}+(u v)_{X L}$. 
Moreover, for any of the bilinear operators $(u v)_{*}$ defined in (2-1)-(2-2), we denote its symbol (multiplier) by $\mathscr{P}_{*}$. We denote finite sums of these bilinear operators in the obvious way, e.g., $(u v)_{L H+H H}=$ $(u v)_{L H}+(u v)_{H H}$. Henceforth, for simplicity, we replace the nonlinear term $\frac{1}{2} \operatorname{Re} N u$ in (1-9) with $N u$ as in [Guo and Nakanishi 2014], because the complex conjugation here makes no essential difference for our arguments. With these notations, it was shown in [Guo and Nakanishi 2014] that (1-9) is equivalent at least for smooth solutions — to the integral equation

$$
\begin{aligned}
u(t)=S(t) u_{0}-S(t) \Omega(N, u)(0)+\Omega(N, u)(t)-i \int_{0}^{t} S(t-s) \Omega\left(\alpha D|u|^{2}, u\right)(s) d s \\
\quad-i \int_{0}^{t} S(t-s) \Omega(N, N u)(s) d s-i \int_{0}^{t} S(t-s)(N u)_{L H+H H+\alpha L}(s) d s
\end{aligned}
$$

and

$$
\begin{array}{r}
N(t)=W_{\alpha}(t) N_{0}-W_{\alpha}(t) D \tilde{\Omega}(u, u)(0)+D \tilde{\Omega}(u, u)(t)-i \int_{0}^{t} W_{\alpha}(t-s) \alpha D(u \bar{u})_{H H+\alpha L+L \alpha} d s \\
-i \int_{0}^{t} W_{\alpha}(t-s)(D \tilde{\Omega}(N u, u)+D \tilde{\Omega}(u, N u))(s) d s,
\end{array}
$$

where $\Omega$ and $\tilde{\Omega}$ are the bilinear Fourier multiplication operators

$$
\begin{aligned}
& \Omega(f, g)=\mathscr{F}^{-1} \int \mathscr{P}_{X L} \frac{\hat{f}(\xi-\eta) \hat{g}(\eta)}{-|\xi|^{2}+\alpha|\xi-\eta|+|\eta|^{2}} d \eta, \\
& \tilde{\Omega}(f, g)=\mathscr{F}^{-1} \int \mathscr{P}_{X L+L X} \frac{\alpha \hat{f}(\xi-\eta) \hat{\bar{g}}(\eta)}{|\xi-\eta|^{2}-|\eta|^{2}-\alpha|\xi|} d \eta .
\end{aligned}
$$

The equations after normal form reduction can be written as

$$
\begin{aligned}
\left(i \partial_{t}+D^{2}\right)(u-\Omega(N, u)) & =(N u)_{L H+H H+\alpha L}+\Omega\left(\alpha D|u|^{2}, u\right)+\Omega(N, N u), \\
\left(i \partial_{t}+\alpha D\right)(N-D \tilde{\Omega}(u, u)) & =\alpha D|u|_{H H+\alpha L+L \alpha}^{2}+D \tilde{\Omega}(N u, u)+D \tilde{\Omega}(u, N u) .
\end{aligned}
$$

2B. Function spaces and Strichartz estimates. Let $s, l \in \mathbb{R}$ and $1 \leq p, q \leq \infty$. We use $B_{p, q}^{s}, \dot{B}_{p, q}^{s}$ to denote the standard Besov space, with norms

$$
\|f\|_{B_{p, q}^{s}}=\left\|P_{\leq 0} f\right\|_{p}+\left(\sum_{k=1}^{\infty} 2^{k s q}\left\|P_{k} f\right\|_{p}^{q}\right)^{\frac{1}{q}}, \quad\|f\|_{\dot{B}_{p, q}^{s}}=\left(\sum_{k=-\infty}^{\infty} 2^{k s q}\left\|P_{k} f\right\|_{p}^{q}\right)^{\frac{1}{q}}
$$

with obvious modifications if $q=\infty$, and we simply write $B_{p}^{s}=B_{p, 2}^{s}, \dot{B}_{p}^{s}=\dot{B}_{p, 2}^{s}$.

For exponents $s \leq l+1$, we use the resolution spaces

$$
\begin{aligned}
& u \in X^{s}:=C\left(\mathbb{R} ; H^{s}\left(\mathbb{R}^{4}\right)\right) \cap L^{\infty}\left(\mathbb{R} ; H^{s}\left(\mathbb{R}^{4}\right)\right) \cap L^{2}\left(\mathbb{R} ; B_{4}^{s}\left(\mathbb{R}^{4}\right)\right), \\
& N \in Y^{l}:=C\left(\mathbb{R} ; H^{l}\left(\mathbb{R}^{4}\right)\right) \cap L^{\infty}\left(\mathbb{R} ; H^{l}\left(\mathbb{R}^{4}\right)\right) .
\end{aligned}
$$


For any Banach function space $Z$ on $\mathbb{R}^{1+4}$ and any interval $I \subset \mathbb{R}$, the restriction of $Z$ onto $I$ is denoted by $Z(I)$. For example,

$$
X^{s}([0, T])=C\left([0, T] ; H^{s}\left(\mathbb{R}^{4}\right)\right) \cap L^{2}\left((0, T) ; B_{4}^{s}\left(\mathbb{R}^{4}\right)\right) .
$$

We will use the following well-known Strichartz estimates for the wave and the Schrödinger equation in dimension $d=4$ :

Lemma 2.1 (Strichartz estimates; see [Keel and Tao 1998]). For any $s \in \mathbb{R}$ and any functions $\phi(x)$ and $f(t, x)$, we have

$$
\begin{aligned}
\|S(t) \phi\|_{L_{t}^{\infty} H_{x}^{s} \cap L_{t}^{2} B_{4}^{s}} & \lesssim\|\phi\|_{H^{s}}, \\
\left\|\int_{0}^{t} S(t-s) f(s) d s\right\|_{L_{t}^{\infty} L_{x}^{2} \cap L_{t}^{2} B_{4}^{0}} & \lesssim\|f\|_{L_{t}^{1} L_{x}^{2}+L_{t}^{2} B_{4 / 3}^{0},}, \\
\left\|W_{\alpha}(t) \phi\right\|_{L_{t}^{\infty} L_{x}^{2} \cap L_{t}^{2} \dot{B}_{6}^{-5 / 6}} & \lesssim\|\phi\|_{L^{2}}, \\
\left\|\int_{0}^{t} W_{\alpha}(t-s) f(s) d s\right\|_{L_{t}^{\infty} L_{x}^{2} \cap L_{t}^{2} \dot{B}_{6}^{-5 / 6}} & \lesssim\|f\|_{L_{t}^{1} L_{x}^{2} .}
\end{aligned}
$$

2C. Multilinear estimates for quadratic and cubic terms. Next, we prove multilinear estimates for the nonlinear terms in (2-5) in the Besov spaces of $x \in \mathbb{R}^{4}$. For $t$, only Hölder's inequalities will be used, which need no explanation. In the following, we ignore the dependence of constants on $(s, l)$, but distinguish by $C(K)$ when it is not uniform for $K$. The main tools are Littlewood-Paley theory and certain Coifman-Meyer-type bilinear Fourier multiplier estimates. Roughly speaking, the multipliers $\Omega$ and $\tilde{\Omega}$ act like

$$
\Omega(f, g) \sim D^{-1}\langle D\rangle^{-1}(f g)_{X L} \quad \text { and } \quad \tilde{\Omega}(f, g) \sim D^{-1}\langle D\rangle^{-1}(f \bar{g})_{X L+L X},
$$

in product estimates in the Besov spaces. Hence the proof is reduced to usual computation of exponents as in the paraproduct. We only sketch the proof.

Lemma 2.2 (quadratic terms). Let $K \geq 5$.

(1) Assume that $s, l \geq 0$. Then, for any $N(x)$ and $u(x)$,

$$
\begin{aligned}
\left\|(N u)_{L H+\alpha L}\right\|_{B_{4 / 3}^{s}} & \lesssim\|N\|_{H^{l}}\|u\|_{B_{4}^{s}}, \\
\left\|(N u)_{H H}\right\|_{B_{4 / 3}^{s}} & \lesssim C(K)\|N\|_{H^{l}}\|u\|_{B_{4}^{s}} .
\end{aligned}
$$

(2) Assume $0 \leq l+1 \leq 2 s$. Then, for any $u(x)$ and $v(x)$,

$$
\begin{aligned}
\left\|D(u v)_{H H}\right\|_{H^{l}} & \lesssim C(K)\|u\|_{B_{4}^{s}}\|v\|_{B_{4}^{s}}, \\
\left\|D(u v)_{\alpha L+L \alpha}\right\|_{H^{l}} & \lesssim\|u\|_{B_{4}^{s}}\|v\|_{B_{4}^{s}} .
\end{aligned}
$$

Proof. The estimates above follow directly from Bony's paraproduct and Hölder's inequality. For example,

$$
\left\|P_{k}(N u)_{L H}\right\|_{L^{4 / 3}} \lesssim \sum_{j=k-2}^{k+2}\left\|\left(P_{\leq j-K} N\right)\left(P_{j} u\right)\right\|_{L^{4 / 3}} \lesssim \sum_{j=k-2}^{k+2}\|N\|_{L^{2}}\left\|P_{j} u\right\|_{L^{4}}
$$


Then we sum up the squares with respect to $k$. The other estimates follow similarly. This argument loses the summability for $H H$ at the 0 regularity ( $s=l=0$ for (1) and $s=l+1=0$ for (2)), but then we can simply use Hölder in $x$ together with the embeddings $B_{p}^{0} \subset L^{p}$ and $L^{p^{\prime}} \subset B_{p^{\prime}}^{0}$ for $2 \leq p \leq \infty$.

Similarly to [Guo et al. 2013, Lemma 4.4; Guo et al. 2014b, Lemma 4.4], we will exploit in the proof of local well-posedness and persistence of regularity that the boundary contributions, as well as cubic terms, can be made small by choosing $K \geq 5$ large.

Lemma 2.3 (boundary terms). There exist $\theta_{j}(s, l) \geq 0$ such that, for all $K \geq 5$ and for any $N(x), u(x)$ and $v(x)$, we have the following:

(1) If $l \geq \max (0, s-2)$ and $(s, l) \neq(2,0)$,

$$
\|\Omega(N, u)\|_{H^{s}} \lesssim 2^{-\theta_{1} K}\|N\|_{H^{l}}\|u\|_{H^{s}}, \quad \theta_{1}>0 \text { for } s<l+2 .
$$

(2) If $l \leq \min (2 s-1, s+1)$ and $(s, l) \neq(2,3)$,

$$
\|D \tilde{\Omega}(u, v)\|_{H^{l}} \lesssim 2^{-\theta_{2} K}\|u\|_{H^{s}}\|v\|_{H^{s}}, \quad \theta_{2}>0 \text { for } l<s+1 .
$$

(3) If $l \geq \min (0, s-1)$ and $(s, l) \neq(1,0)$,

$$
\|\Omega(N, u)\|_{B_{4}^{s}} \lesssim 2^{-\theta_{3} K}\|N\|_{H^{l}}\|u\|_{B_{4}^{s}}, \quad \theta_{3}>0 \text { for } s<l+1 .
$$

(4) If $l \leq \min \left(2 s-\frac{1}{2}, s+\frac{3}{2}\right)$ and $(s, l) \neq\left(2, \frac{7}{2}\right)$,

$$
\left\|\langle D\rangle^{l} \tilde{\Omega}(u, v)\right\|_{\dot{B}_{6}^{1 / 6}} \lesssim 2^{-\theta_{4} K}\left[\|u\|_{B_{4}^{s}}\|v\|_{H^{s}}+\|v\|_{B_{4}^{s}}\|u\|_{H^{s}}\right], \quad \theta_{4}>0 \text { for } l<s+\frac{3}{2} .
$$

Proof. Since they are all straightforward, we prove only (2-14)-(2-15), leaving (2-12)-(2-13) to the reader. By [Guo and Nakanishi 2014, Lemma 3.5] and using (2-8) with Bernstein, we have

$$
\begin{aligned}
\left\|P_{k}\langle D\rangle D \Omega\left(P_{k_{0}} N, P_{k_{1}} u\right)\right\|_{L_{x}^{p}} & \lesssim\left\|P_{k_{0}} N\right\|_{L_{x}^{p_{0}}\left\|P_{k_{1}} u\right\|_{L_{x}^{p_{1}}}} \\
& \lesssim 2^{4 k_{0}\left(1 / p_{0}-1 / q_{0}\right)+4 k_{1}\left(1 / p_{1}-1 / q_{1}\right)}\left\|P_{k_{0}} N\right\|_{L_{x}^{q_{0}}}\left\|P_{k_{1}} u\right\|_{L_{x}^{q_{1}}}
\end{aligned}
$$

for any $k, k_{0}, k_{1} \in \mathbb{Z}$ and any $p, p_{0}, p_{1}, q_{0}, q_{1} \in[1, \infty]$ satisfying $1 / p=1 / p_{0}+1 / p_{1}$ and $q_{j} \leq p_{j}$. The same estimate holds for the bilinear operator $\tilde{\Omega}$. For the low frequency part, say if $k_{1} \leq k_{0}-K$, we can replace $P_{k_{1}}$ with $P_{\leq k_{1}}$. The above with $\left(p, p_{0}, p_{1}, q_{0}, q_{1}\right)=(4,4, \infty, 2,4)$ and the $H L$ restriction $\left|k-k_{0}\right| \leq 1$ in $\Omega$ yields

$$
\|\Omega(N, u)\|_{B_{4}^{s}} \lesssim\left\|2^{k^{+}(s-1-l)}\right\| P_{k} N\left\|_{H^{l}} \sum_{k_{1} \leq k-K} 2^{k_{1}-k_{1}^{+} s}\right\| P_{k_{1}} u\left\|_{B_{4}^{s}}\right\|_{l_{k}^{2}},
$$

where $k^{+}:=\max (k, 0)$, using $P_{\leq 0} B_{p}^{s} \subset \dot{B}_{p, \infty}^{0}$ for the lower frequency component. The summation over $k_{1} \leq k-K$ is bounded by

$$
\begin{cases}2^{k-K} & \text { if } k \leq K \\ 2^{(1-s)^{+}(k-K)} & \text { if } k>K, s \neq 1 \\ k-K & \text { if } k>K, s=1\end{cases}
$$


This and $\left\|P_{k} N\right\|_{H^{l}} \in \ell_{k}^{2}$ lead to (2-14), with the small factor $2^{-\theta_{3} K}$ for $s<1$ and for $1 \leq s<l+1$. The conditions $l \geq 0$ and $l \geq s-1$ ensure uniform boundedness of the coefficient after the summation for $s<1$ and for $s>1$, respectively, while the endpoint $(s, l)=(1,0)$ is excluded due to the logarithmic growth at $s=1$. Similarly, with $\left(p, p_{0}, p_{1}, q_{0}, q_{1}\right)=(6,6, \infty, 4,2)$, we have

$$
\left\|P_{k}\langle D\rangle^{l} \tilde{\Omega}(u, v)_{H L}\right\|_{\dot{B}_{6}^{1 / 6}} \lesssim 2^{k^{+}(l-1-s)-k / 2} \sum_{k_{1} \leq k-K} 2^{2 k_{1}-k_{1}^{+} s}\left\|P_{k} u\right\|_{B_{4}^{s}}\left\|P_{k_{1}} v\right\|_{H^{s}}
$$

Using this and $\left\|P_{k} u\right\|_{B_{4}^{s}} \in \ell_{k \geq 0}^{2}$ lead to (2-15), with the small factor for $s<2$ and for $2 \leq s<l-\frac{3}{2}$.

Lemma 2.4 (cubic terms). There exist $\theta_{j}(s, l) \geq 0$ such that, for all $K \geq 5$ and for any $M(x), N(x), u(x)$, $v(x)$ and $w(x)$, we have the following:

(1) If $s \geq \frac{1}{2}$, then $\theta_{1}>0$ and

$$
\|\Omega(D(u v), w)\|_{H^{s}} \lesssim 2^{-\theta_{1} K}\left[\|u\|_{H^{s}}\|v\|_{B_{4}^{1 / 2}}+\|v\|_{H^{s}}\|u\|_{B_{4}^{1 / 2}}\right]\|w\|_{B_{4}^{1 / 2}}
$$

(2) If $l \geq 0,-l<s \leq l+2, s \leq 2 l+1$, and $(s, l) \neq(1,0)$,

$$
\|\Omega(M, N u)\|_{B_{4 / 3}^{s}} \lesssim 2^{-\theta_{2} K}\|M\|_{H^{l}}\|N\|_{H^{l}}\|u\|_{B_{4}^{s}}, \quad \theta_{2}>0 \text { for } s<l+2 .
$$

(3) If $s \geq \frac{1}{2},-s<l \leq s+1, l \leq 2 s$, and $(s, l) \neq(1,2)$,

$$
\|D \tilde{\Omega}(N u, v)\|_{H^{l}}+\|D \tilde{\Omega}(v, N u)\|_{H^{l}} \lesssim 2^{-\theta_{3} K}\|N\|_{H^{l}}\|u\|_{B_{4}^{s}}\|v\|_{B_{4}^{s}}, \quad \theta_{3}>0 \text { for } l<s+1 .
$$

Proof. For (2-20), we can use a standard product inequality for $s \geq \frac{1}{2}$ :

$$
\|u v\|_{B_{8 / 5}^{s}} \lesssim\|u\|_{H^{s}}\|v\|_{B_{4}^{1 / 2}}+\|v\|_{H^{s}}\|u\|_{B_{4}^{1 / 2}}
$$

which easily follows using $B_{4}^{1 / 2} \subset L^{8}$, e.g., by the paraproduct calculus. Putting $f:=u v$ we obtain, from (2-16) with $\left(p, p_{0}, p_{1}, q_{0}, q_{1}\right)=\left(2,2, \infty, \frac{8}{5}, 4\right)$,

$$
\left\|P_{k} \Omega(D f, w)\right\|_{H^{s}} \lesssim 2^{k / 2-k^{+}} \sum_{k_{1} \leq k-K} 2^{k_{1}-k_{1}^{+} / 2}\|f\|_{B_{8 / 5}^{s}}\|w\|_{B_{4}^{1 / 2}},
$$

which leads to (2-20) with a small factor, in the same way as in the previous lemma.

For (2-21) and (2-22), we can use a standard product inequality:

$$
\sigma \leq \min (s, l, s+l-1) \Longrightarrow\|N u\|_{H^{\sigma}} \lesssim\|N\|_{H^{l}}\|u\|_{B_{4}^{s}}
$$

which holds for $s+l>0$ unless $s=1$ and $\sigma=l$. Putting $g:=N u$ we obtain, from (2-16) with $\left(p, p_{0}, p_{1}, q_{0}, q_{1}\right)=\left(\frac{4}{3}, 2,4,2,2\right)$,

$$
\left\|P_{k} \Omega(M, g)\right\|_{B_{4 / 3}^{s}} \lesssim \sum_{k_{1} \leq k-K} 2^{k^{+}(s-1-l)-k+k_{1}-k_{1}^{+} \sigma}\left\|P_{k} M\right\|_{H^{l}}\left\|P_{k_{1}} g\right\|_{H^{\sigma}} .
$$


First, the low frequency part $k \leq 0$ is bounded using Young on $\mathbb{Z}$ by

$$
\begin{aligned}
\left\|P_{\leq 0} \Omega(M, g)\right\|_{B_{4 / 3}^{s}} \lesssim\left\|P_{k} \Omega(M, g)\right\|_{\ell_{k \leq 0}^{1} L_{x}^{4 / 3}} & \lesssim\left\|P_{k} M\right\|_{\ell_{k_{\leq}^{2}}^{2} H_{x}^{l}}\left\|\sum_{k_{1} \leq k-K} 2^{-k+k_{1}}\right\| P_{k_{1}} g\left\|_{H_{x}^{\sigma}}\right\|_{\ell_{k \leq 0}^{2}} \\
& \lesssim 2^{-K}\|M\|_{H^{l}}\|g\|_{H^{\sigma}} .
\end{aligned}
$$

For $0<k \leq K$, the summation over $k_{1}$ is bounded by $2^{k(s-l-1)-K}\left\|P_{k} M\right\|_{H^{l}} \in \ell_{k}^{2}$ with the small factor for $s<l+2$. For $K<k$, it is bounded by

$$
\begin{cases}2^{k(s-1-l-\sigma)} 2^{-K(1-\sigma)} & \text { if } \sigma<1 \\ 2^{k(s-2-l)} & \text { if } \sigma>1 .\end{cases}
$$

The case $\sigma<1$ is fine if $\sigma=l$ by $s \leq 2 l+1$, if $\sigma \leq s+1$ by $l \geq 0$, and if $\sigma=s+l-1$ by $l \geq 0$. In the critical case $s=1$ for the product inequality, we have $s<2 l+1$ and $l>0$ by the exclusion $(s, l) \neq(1,0)$, so that we can choose $\sigma=l-\varepsilon$. The case $\sigma>1$ is fine by $s \leq l+2$. Then the only remaining case is $(s, l)=(3,1)$, where we are forced to choose $\sigma=1$; then we should replace (2-26) for $k>K$ with

$$
\left\|P_{k} \Omega(M, g)\right\|_{B_{4 / 3}^{s}} \lesssim 2^{k(s-2-l)}\left\|P_{k} M\right\|_{H^{l}}\left\|P_{\leq k-K} g\right\|_{H^{1}},
$$

which is bounded using $\left\|P_{k} M\right\|_{H^{l}} \in \ell_{k}^{2}$. Thus we obtain (2-21).

Similarly, from (2-16) with $\left(p, p_{0}, p_{1}, q_{0}, q_{1}\right)=(2,2, \infty, 2,4)$, we have

$$
\left\|P_{k} D \tilde{\Omega}(g, v)_{H L}\right\|_{H^{l}}+\left\|P_{k} D \tilde{\Omega}(v, g)_{L H}\right\|_{H^{l}} \lesssim \sum_{k_{1} \leq k-K} 2^{k^{+}(l-1-\sigma)+k_{1}-k_{1}^{+} s}\left\|P_{k} g\right\|_{H^{\sigma}}\left\|P_{k_{1}} v\right\|_{B_{4}^{s}},
$$

for which the low frequencies $k \leq K$ are easily bounded using the factor $2^{k_{1}}$, while for $k>K$ the summation is bounded by

$$
\begin{cases}2^{k(l-s-\sigma)} 2^{-K(1-s)} & \text { if } s<1, \\ 2^{k(l-1-\sigma)}(k-K) & \text { if } s=1, \\ 2^{k(l-1-\sigma)} & \text { if } s>1 .\end{cases}
$$

The case $s<1$ is fine if $\sigma=s$ by $l \leq 2 s$, and if $\sigma=s+l-1$ by $s \geq \frac{1}{2}$. The case $s>1$ is fine if $\sigma=s$ by $l \leq s+1$, and obviously if $\sigma=l$. The critical case $s=1$ is also fine, as none of the conditions is on the boundary, thanks to $(s, l) \neq(1,2)$.

For the other $H L$ interaction, choosing $\left(p, p_{0}, p_{1}, q_{0}, q_{1}\right)=(2,4,4,2,4)$ we have

$$
\left\|P_{k} D \tilde{\Omega}(g, v)_{L H}\right\|_{H^{l}}+\left\|P_{k} D \tilde{\Omega}(v, g)_{H L}\right\|_{H^{l}} \lesssim \sum_{k_{1} \leq k-K} 2^{k^{+}(l-1-s)+k_{1}-k_{1}^{+} \sigma}\left\|P_{k} v\right\|_{B_{4}^{s}}\left\|P_{k_{1}} g\right\|_{H^{\sigma}},
$$

which is also easy for $k \leq K$. For $k>K$, the summation is bounded by

$$
\begin{cases}2^{k(l-\sigma-s)} 2^{-K(1-\sigma)} & \text { if } \sigma<1 \\ 2^{k(l-1-s)} & \text { if } \sigma>1\end{cases}
$$

The case $\sigma<1$ is the same as the case $s<1$ in (2-31). The case $\sigma>1$ is OK by $l \leq s+1$. When $l=s+1 \geq \frac{3}{2}$, we can choose $\sigma=\min (s, l, s+l-1)=s \neq 1$ thanks to $(s, l) \neq(1,2)$. In the critical 
case $s=1$, we can choose $\sigma<\min (s, l, s+l-1) \leq 1$ such that $l-s-\sigma<0$, since $l<2 s=2$. This concludes the proof of (2-22).

\section{Small data scattering for $s \leq l+1$}

Using the multilinear estimates in the previous section, it is now easy to obtain global well-posedness and scattering for small initial data in $H^{s} \times H^{l}$ in the range (1-11) under $s \leq l+1$. In Section 5 we will show that we only need smallness in $H^{1 / 2} \times L^{2}$ for all regularities by a persistence of regularity argument. Fix $K=5$. As in [Guo and Nakanishi 2014, Section 4], for fixed initial data $\left(u_{0}, N_{0}\right) \in H^{s} \times H^{l}$ we define a mapping $(u, N) \mapsto\left(u^{\prime}, N^{\prime}\right)=\Phi_{u_{0}, N_{0}}(u, N)$ by the right-hand sides of the equations (2-3)-(2-4). Then, for small initial data $\left(u_{0}, N_{0}\right)$, we see that $\Phi_{u_{0}, N_{0}}$ is a contraction in a small ball around 0 of $X^{s} \times Y^{l}$. Indeed, from the estimates in the previous section, we obtain

$$
\begin{aligned}
\left\|u^{\prime}\right\|_{X^{s}} & \lesssim\left\|u_{0}\right\|_{H^{s}}+\|N\|_{Y^{l}}\|u\|_{X^{s}}+\|u\|_{X^{s}}^{3}+\|N\|_{Y^{l}}^{2}\|u\|_{X^{s}} \\
\left\|N^{\prime}\right\|_{Y^{l}} & \lesssim\left\|N_{0}\right\|_{H^{l}}+\|u\|_{X^{s}}^{2}+\|N\|_{Y^{l}}\|u\|_{X^{s}}^{2}
\end{aligned}
$$

where we need $s \leq l+1$ in using (2-14) for $\Omega(N, u)$. By the contraction mapping principle, we have a unique solution in a small ball in $X^{s} \times Y^{l}$, and the Lipschitz continuity of the solution map $H^{s} \times H^{l} \rightarrow X^{s} \times Y^{l}$ follows from the standard argument.

Now we derive scattering for $(u, N)$ in $H^{s} \times H^{l}$, assuming we have $(s, l)$ satisfying $(1-11)$, that $(u, N)$ is in $X^{1 / 2} \times Y^{0}$ with small norm, and the scattering of the transformed variables, namely, for

$$
\Psi(u, N):=(u-\Omega(N, u), N-D \tilde{\Omega}(u, u))
$$

there exist $\left(u_{ \pm}, N_{ \pm}\right) \in H^{s} \times H^{l}$ with small norm in $H^{1 / 2} \times L^{2}$ such that

$$
\Psi(u, N)-\left(S(t) u_{ \pm}, W_{\alpha}(t) N_{ \pm}\right) \rightarrow 0 \quad \text { in } H^{s} \times H^{l} \quad(t \rightarrow \pm \infty) .
$$

In the current case $s \leq l+1$, the latter assumption, (3-3), obviously holds in view of the fact that $(u, N) \in X^{s} \times Y^{l}$ and the Strichartz estimate with the global bounds on the nonlinear terms.

The bilinear estimate for the normal form in Lemma 2.3 implies that the above transform $\Psi$ is invertible for small data in $H^{1 / 2} \times L^{2}$ and bi-Lipschitz. More precisely, for any $\left(u^{\prime}, N^{\prime}\right) \in H^{1 / 2} \times L^{2}$, the inverse image $\Psi^{-1}\left(u^{\prime}, N^{\prime}\right)$ is the fixed points of the map

$$
(u, N) \mapsto \Psi_{u^{\prime}, N^{\prime}}(u, N):=\left(u^{\prime}+\Omega(N, u), N^{\prime}+D \tilde{\Omega}(u, u)\right) .
$$

Lemma 2.3 implies that $\Psi_{u^{\prime}, N^{\prime}}$ is a contraction in a small ball of $H^{1 / 2} \times L^{2}$ if $\left(u^{\prime}, N^{\prime}\right)$ is small, hence the unique small $(u, N) \in \Psi^{-1}\left(u^{\prime}, N^{\prime}\right)$ is given by the iteration

$$
(u, N)=\lim _{k \rightarrow \infty}\left(\Psi_{u^{\prime}, N^{\prime}}\right)^{k}(0,0) .
$$

By (3-3), we get

$$
(u, N)-\Psi^{-1}\left(S(t) u_{ \pm}, W_{\alpha}(t) N_{ \pm}\right) \rightarrow 0 \quad \text { in } H^{1 / 2} \times L^{2} \quad(t \rightarrow \pm \infty) .
$$


To show the scattering for $(u, N)$, it suffices to show

$$
\Psi^{-1}\left(S(t) u_{ \pm}, W_{\alpha}(t) N_{ \pm}\right) \rightarrow\left(S(t) u_{ \pm}, W_{\alpha}(t) N_{ \pm}\right) \quad \text { in } H^{1 / 2} \times L^{2} \quad(t \rightarrow \pm \infty) .
$$

By the construction of the inverse, we get

$$
\left(u_{ \pm}^{n}(t), N_{ \pm}^{n}(t)\right) \rightarrow \Psi^{-1}\left(S(t) u_{ \pm}, W_{\alpha}(t) N_{ \pm}\right) \quad \text { in } L_{t}^{\infty}\left(H^{1 / 2} \times L^{2}\right) \quad(n \rightarrow \infty),
$$

where $\left(u_{ \pm}^{0}, N_{ \pm}^{0}\right)=(0,0)$, and, for $n=1,2, \ldots$,

$$
\begin{aligned}
u_{ \pm}^{n+1} & =S(t) u_{ \pm}+\Omega\left(N_{ \pm}^{n}, u_{ \pm}^{n}\right), \\
N_{ \pm}^{n+1} & =W_{\alpha}(t) N_{ \pm}+D \tilde{\Omega}\left(u_{ \pm}^{n}, u_{ \pm}^{n}\right) .
\end{aligned}
$$

Thus, to show (3-6), it suffices to show for any $n$ that

$$
\left(u_{ \pm}^{n}(t), N_{ \pm}^{n}(t)\right) \rightarrow\left(S(t) u_{ \pm}, W_{\alpha}(t) N_{ \pm}\right) \quad \text { in } H^{1 / 2} \times L^{2} \quad(t \rightarrow \pm \infty),
$$

for which, by induction on $n$ and bilinear estimates, it suffices to show

$$
\left(\Omega\left(N_{F}, u_{F}\right), D \tilde{\Omega}\left(u_{F}, u_{F}\right)\right) \rightarrow 0 \quad \text { in } H^{s} \times H^{l} \quad(t \rightarrow \pm \infty)
$$

for all free solutions $\left(u_{F}, N_{F}\right)$ in $H^{s} \times H^{l}$. The density argument with the bilinear estimate allows us to restrict to the case $u_{F}(0), N_{F}(0) \in C_{0}^{\infty}\left(\mathbb{R}^{4}\right)$; then the above is almost obvious, by the dispersive decay of $S(t)$ and $W_{\alpha}(t)$ (we omit the details).

For higher regularity, $(s, l) \neq\left(\frac{1}{2}, 0\right)$, we do not have smallness in $H^{s} \times H^{l}$, so we should replace Lemma 2.3 with the estimates

$$
\begin{aligned}
\|\Omega(N, u)\|_{H^{s}} & \lesssim\|N\|_{H^{l}}\|u\|_{B_{u}}, \\
\|\Omega(N, u)\|_{B_{u}} & \lesssim\|N\|_{B_{N}}\|u\|_{B_{u}}, \\
\left\|D \tilde{\Omega}(u, u)_{H L}\right\|_{H^{l}} & \lesssim\|u\|_{H^{s}}\|u\|_{B_{u}},
\end{aligned}
$$

where the Besov spaces $B_{u}$ and $B_{N}$ are defined by

$$
B_{u}:=B_{p}^{s-\varepsilon}, \quad B_{N}:=B_{p}^{l-\varepsilon}, \quad \frac{1}{p}=\frac{1}{2}-\frac{\varepsilon}{4}
$$

for some small $\varepsilon>0$ such that $H^{s} \times H^{l} \subset B_{u} \times B_{N}$ by the sharp Sobolev embedding. The estimates (3-10) imply that $\Psi_{u^{\prime}, N^{\prime}}$ is a contraction with respect to the equivalent norm

$$
\|(u, N)\|_{Z}:=\|u\|_{H^{s}}+\|N\|_{H^{l}}+\delta^{-2}\|u\|_{B_{u}}
$$

for $0<\delta \ll 1$ on the closed set

$$
F:=\left\{(u, N) \in H^{s} \times H^{l} \mid\|(u, N)\|_{Z} \leq 1 / \delta,\|N\|_{B_{N}} \leq \delta,\|u\|_{B_{u}} \leq \delta^{3}\right\}
$$

provided that $2\left(u^{\prime}, N^{\prime}\right) \in F$. Indeed, (3-10) yields, for any $(u, N) \in F$,

$$
\|(\Omega(N, u), D \tilde{\Omega}(u, u))\|_{H^{s} \times H^{l}} \lesssim \delta^{2}, \quad\|\Omega(N, u)\|_{B_{u}} \lesssim \delta^{4},
$$


hence $\|(\Omega(N, u), D \tilde{\Omega}(u, u))\|_{Z} \lesssim \delta^{2}$ and $\Psi_{u^{\prime}, N^{\prime}}(u, N) \in F$. For the difference, we have from (3-10), for any $(v, M) \in H^{s} \times H^{l}$,

$$
\begin{aligned}
\left\|\left(\Omega(N, v), D \tilde{\Omega}(u, v)_{H L}\right)\right\|_{H^{s} \times H^{l}} & \lesssim\|(u, N)\|_{H^{s} \times H^{l}}\|v\|_{B_{u}} \lesssim \delta\|(v, M)\|_{Z}, \\
\left\|\left(\Omega(M, u), D \tilde{\Omega}(v, u)_{H L}\right)\right\|_{H^{s} \times H^{l}} & \lesssim\|(v, M)\|_{H^{s} \times H^{l}}\|u\|_{B_{u}} \lesssim \delta^{3}\|(v, M)\|_{Z}, \\
\|\Omega(N, v)+\Omega(M, u)\|_{B_{u}} & \lesssim\|N\|_{B_{N}}\|v\|_{B_{u}}+\|M\|_{B_{N}}\|u\|_{B_{u}} \lesssim \delta^{3}\|(v, M)\|_{Z} .
\end{aligned}
$$

Since the scattering of $\Psi(u, N)$ implies $\|\Psi(u, N)\|_{B_{u} \times B_{N}} \rightarrow 0$ as $t \rightarrow \infty$, choosing $\delta>0$ small enough ensures that $2 \Psi(u, N) \in F$ for large $t$. Then $(u, N)$ given by (3-5) is the same as the fixed point in $F$. Since we can take $\delta>0$ arbitrarily small, (3-14) implies that $\|(u, N)-\Psi(u, N)\|_{H^{s} \times H^{l}} \rightarrow 0$ as $t \rightarrow \infty$, hence the scattering of $(u, N)$ in $H^{s} \times H^{l}$.

Since all the estimates are uniform and global in time, the same argument works for the final state problem, namely to find the solution for a prescribed (small) scattering data at $t=\infty$. Thus we obtain:

Proposition 3.1. Let $(s, l) \in \mathbb{R}^{2}$ satisfy (1-11), $s \leq l+1$, and $(s, l) \neq(1,0)$. Then there exists $\varepsilon_{1}=\varepsilon_{1}(s, l)>0$ such that, for any $\left(u_{0}, N_{0}\right) \in H^{s}\left(\mathbb{R}^{4}\right) \times H^{l}\left(\mathbb{R}^{4}\right)$ satisfying $\left\|\left(u_{0}, N_{0}\right)\right\|_{H^{s} \times H^{l}} \leq \varepsilon_{1}$, there exists a unique global solution $(u, N) \in X^{s} \times Y^{l}$ of (1-9). Moreover, there exists $\left(u^{+}, N^{+}\right) \in H^{s} \times H^{l}$ such that

$$
\lim _{t \rightarrow \infty}\left\|u(t)-S(t) u^{+}\right\|_{H_{x}^{s}}+\left\|N(t)-W_{\alpha}(t) N^{+}\right\|_{H_{x}^{l}}=0
$$

Conversely, for any $\left(u^{+}, N^{+}\right) \in H^{s} \times H^{l}$ with $\left\|\left(u^{+}, N^{+}\right)\right\|_{H^{s} \times H^{l}} \leq \varepsilon_{1}$, there exists a unique solution $(u, N) \in X^{s} \times Y^{l}$ satisfying (3-16). Both the maps $\left(u_{0}, N_{0}\right) \mapsto(u, N)$ and $\left(u^{+}, N^{+}\right) \mapsto(u, N)$ are Lipschitz continuous from the $\varepsilon_{1}$-ball into $X^{s} \times Y^{l}$.

The uniqueness without the smallness is proved in the next section. For the question of whether $(u, N)$ obtained above really solves (1-9) before the normal form, see Remark 5.3.

\section{Large data local well-posedness for $s<l+1$}

For large data, the proof in the previous section does not immediately work, in particular at the endpoint $(s, l)=\left(\frac{1}{2}, 0\right)$. The main difficulty is the lack of flexibility in the choice of the Strichartz norm for the boundary term and the bilinear term $(N u)_{L H}$. More precisely, $L_{t}^{\infty} H_{x}^{l}$ is the only choice among the Strichartz norms of $W_{\alpha}(t)$ for $N$ to estimate $\Omega(N, u)$ in $L_{t}^{\infty} H_{x}^{s}$ and to avoid losing regularity in $(N u)_{L H}$. For the former term, we can play with the frequency gap parameter $K$ in the normal form to extract a small factor. For the latter term, we use the following:

Lemma 4.1. Let $0<T \leq \infty$ and $N \in C\left([0, T) ; L^{2}\left(\mathbb{R}^{4}\right)\right)$. Suppose that $W_{\alpha}(-t) N(t)$ is strongly convergent in $L_{x}^{2}$ as $t \rightarrow T-0$. Then, for any $\varepsilon>0$, there exists a finite increasing sequence $0=T_{0}<T_{1}<\cdots<T_{n+1}=T$ such that

$$
\|N\|_{\left(L_{t}^{\infty} L_{x}^{2}+L_{t}^{2} L_{x}^{4}\right)\left(T_{j}, T_{j+1}\right)}<\varepsilon
$$

for each $j=0, \ldots, n$. 
Note that the $L_{t}^{2} L_{x}^{4}$ norm is not controlled by the Strichartz estimate for $W_{\alpha}(t)$, but it is bounded for nice initial data. The case $T=\infty$ will be used for large data scattering. For $T<\infty$, the assumption on $N$ is equivalent to $N \in C\left([0, T] ; L^{2}\right)$.

Proof. Put $N^{+}:=\lim _{t \rightarrow T-0} W_{\alpha}(-t) N(t) \in L_{x}^{2}$. By the strong convergence, there exists $T^{\prime} \in(0, T)$ such that $\sup _{T^{\prime} \leq t<T}\left\|N(t)-W_{\alpha}(t) N^{+}\right\|_{L_{x}^{2}}<\frac{1}{4} \varepsilon$. Since $C_{0}^{\infty} \subset L_{x}^{2}$ is dense, there exists $N_{0} \in C_{0}^{\infty}$ such that $\left\|N_{0}-N^{+}\right\|_{L_{x}^{2}}<\frac{1}{4} \varepsilon$. The dispersive decay of $W_{\alpha}(t)$ implies that $W_{\alpha}(t) N_{0} \in L_{t}^{2} L_{x}^{4}(\mathbb{R})$. Define $N^{\prime}$ by

$$
N^{\prime}(t):= \begin{cases}P_{\leq k} N(t) & \text { if } 0 \leq t \leq T^{\prime} \\ W_{\alpha}(t) N_{0} & \text { if } T^{\prime}<t<T\end{cases}
$$

By the above choice of $T^{\prime}$ and $N_{0}$, we have $\left\|N-N^{\prime}\right\|_{L^{\infty}\left(\left(T^{\prime}, T\right) ; L_{x}^{2}\right)}<\frac{1}{2} \varepsilon$. Since $N \in C\left(\left[0, T^{\prime}\right] ; L_{x}^{2}\right)$ and $\left[0, T^{\prime}\right]$ is compact, $N^{\prime}(t) \rightarrow N(t)$ in $L_{x}^{2}$ uniformly on $t \in\left[0, T^{\prime}\right]$ as $k \rightarrow \infty$. Hence, for large $k$, we have $\left\|N-N^{\prime}\right\|_{L^{\infty}\left(\left[0, T^{\prime}\right] ; L_{x}^{2}\right)}<\frac{1}{2} \varepsilon$. Hence,

$$
\left\|N-N^{\prime}\right\|_{L^{\infty}\left([0, T) ; L_{x}^{2}\right)}<\frac{1}{2} \varepsilon, \quad N^{\prime} \in L_{t}^{2}\left([0, T) ; L_{x}^{4}\right) .
$$

Choosing $T_{1}<T_{2}<\cdots<T_{n}$ appropriately ensures that $\left\|N^{\prime}\right\|_{L_{t}^{2} L_{x}^{4}\left(T_{j}, T_{j+1}\right)}<\frac{1}{2} \varepsilon$ for each $j$, then we get the desired estimate.

Now we are ready to prove the local well-posedness for large data in $H^{1 / 2} \times L^{2}$. For any initial data $\left(u_{0}, N_{0}\right) \in H^{1 / 2} \times L^{2}$, let

$$
u_{F}:=S(t)\left(u_{0}-\Omega\left(N_{0}, u_{0}\right)\right), \quad N_{F}:=W_{\alpha}(t)\left(N_{0}-D \tilde{\Omega}\left(u_{0}, u_{0}\right)\right)
$$

and apply Lemma 4.1 to $N_{F}$. Then, for any $\varepsilon>0$, there exists $T>0$ such that

$$
\left\|u_{F}\right\|_{L_{t}^{2} B_{4}^{1 / 2}(0, T)}+\left\|N_{F}\right\|_{L_{t}^{\infty} L_{x}^{2}+L_{t}^{2} L_{x}^{4}(0, T)}<\varepsilon
$$

Putting $\mathscr{H}:=H^{1 / 2} \times L^{2}$ and $m:=\left\|\left(u_{F}(0), N_{F}(0)\right)\right\|_{\mathscr{H}}$, we look for a unique local solution on $(0, T)$ as a fixed point of the map $\Phi_{u_{0}, N_{0}}$ in the closed set

$$
K_{m}^{\varepsilon}:=\left\{(u, N) \in C([0, T] ; \mathcal{H}) \mid\|(u, N)\|_{L_{t}^{\infty}(0, T ; \mathscr{H})} \leq 2 m,\|u\|_{L_{t}^{2} B_{4}^{1 / 2}(0, T)}+\|N\|_{L_{t}^{\infty} L_{x}^{2}+L_{t}^{2} L_{x}^{4}(0, T)} \leq 2 \varepsilon\right\} .
$$

From the multilinear estimates in Section 2, we have

$$
\begin{aligned}
\|\Omega(N, u)\|_{X^{1 / 2}} & \lesssim 2^{-\theta K}\|N\|_{L_{t}^{\infty} L_{x}^{2}}\|u\|_{X^{1 / 2}}, \\
\left\|\Omega\left(D|u|^{2}, u\right)\right\|_{L_{t}^{1} H_{x}^{1 / 2}} & \lesssim 2^{-\theta K}\|u\|_{L_{t}^{\infty} H^{1 / 2}\|u\|_{L_{t}^{2} B_{4}^{1 / 2}}^{2}}, \\
\|\Omega(N, N u)\|_{L_{t}^{2} B_{4 / 3}^{1 / 2}} & \lesssim 2^{-\theta K}\|N\|_{L_{t}^{\infty} L_{x}^{2}}^{2}\|u\|_{L_{t}^{2} B_{4}^{1 / 2}} \\
\|D \tilde{\Omega}(u, u)\|_{L_{t}^{\infty} L_{x}^{2}} & \lesssim 2^{-\theta K}\|u\|_{L_{t}^{\infty} H_{x}^{1 / 2}}^{2}, \\
\|D \tilde{\Omega}(N u, u)\|_{L_{t}^{1} L_{x}^{2}} & \lesssim 2^{-\theta K}\|N\|_{L_{t}^{\infty} L_{x}^{2}}\|u\|_{L_{t}^{2} B_{4}^{1 / 2}}^{2},
\end{aligned}
$$


and the same estimate on $D \tilde{\Omega}(u, N u)$, as well as for the difference. Taking $K$ large makes these estimates contractive. For the remaining two terms,

$$
\begin{aligned}
&\left\|D|u|_{H H+\alpha L+L \alpha}^{2}\right\|_{L_{t}^{1} L_{x}^{2}} \lesssim C(K)\|u\|_{L_{t}^{2} B_{4}^{1 / 2}}^{2}, \\
&\left\|(N u)_{L H+H H+\alpha L}\right\|_{L_{t}^{2} B_{4 / 3}^{1 / 2}+L_{t}^{1} H_{x}^{1 / 2}} \lesssim C(K)\|N\|_{L_{t}^{\infty} L_{x}^{2}+L_{t}^{2} L_{x}^{4}}\|u\|_{L_{t}^{2} B_{4}^{1 / 2}},
\end{aligned}
$$

which is also made contractive on the interval $[0, T]$ by choosing $\varepsilon>0$ small enough that $C(K) \varepsilon \ll 1$ after fixing $K$. Then $\Phi_{u_{0}, N_{0}}$ becomes a contraction on $K_{m}^{\varepsilon}$.

The uniqueness of the solution in the class $X^{1 / 2} \times Y^{0}$ is obtained in the same fashion: Let $\left(u_{j}, N_{j}\right)$ for $j=0,1$ be two solutions in $X^{1 / 2} \times Y^{0}$. For any $\varepsilon>0$, applying Lemma 4.1 we can find $T^{\prime} \in(0, T)$ such that, for $j=0,1$,

$$
\left\|u_{j}\right\|_{L_{t}^{2} B_{4}^{1 / 2}\left(0, T^{\prime}\right)}+\left\|N_{j}\right\|_{L_{t}^{\infty} L_{x}^{2}+L_{t}^{2} L_{x}^{4}\left(0, T^{\prime}\right)}<\varepsilon
$$

so that both the solutions belong to $K_{m}^{\varepsilon}$ on $\left[0, T^{\prime}\right]$, hence $\left(u_{0}, N_{0}\right)=\left(u_{1}, N_{1}\right)$ as long as they are solutions in the above class.

The continuous dependence is also obtained in the same way, because

$$
H^{1 / 2} \times L^{2} \rightarrow X^{1 / 2} \times Y^{0}, \quad\left(u_{0}, N_{0}\right) \mapsto\left(u_{F}, N_{F}\right)
$$

is continuous. Take a strongly convergent sequence of initial data. If the smallness condition (4-5) is satisfied by the limit, then so it is by those sufficiently close to the limit. Then we can estimate the difference from the limit in the same way as above, leading to the strong continuity.

We have worked at the lowest regularity $(s, l)=\left(\frac{1}{2}, 0\right)$, but the same argument works as long as we have the small factor $2^{-\theta K}$, namely for $|s-l|<1$. Thus we obtain:

Proposition 4.2. Let $(s, l) \in \mathbb{R}^{2}$ satisfy (1-11) and $|s-l|<1$. For any $\left(u_{0}, N_{0}\right) \in H^{s}\left(\mathbb{R}^{4}\right) \times H^{l}\left(\mathbb{R}^{4}\right)$, there exists a unique local solution $(u, N) \in\left(X^{s} \times Y^{l}\right)([0, T])$ of $(1-9)$ for some $T>0$, where both $T$ and $(u, N)$ depend continuously on $\left(u_{0}, N_{0}\right)$. More precisely, if $\left(u_{0, n}, N_{0, n}\right) \rightarrow\left(u_{0}, N_{0}\right)$ in $H^{s} \times H^{l}$, then $T_{n} \rightarrow T$ and, for any $0<T^{\prime}<T$, we have $\left\|u_{n}-u\right\|_{X^{s}\left(\left[0, T^{\prime}\right]\right)}+\left\|N_{n}-N\right\|_{Y^{l}\left(\left[0, T^{\prime}\right]\right)} \rightarrow 0$.

\section{Persistence of regularity except for $(s, l)=(1,0)$}

Once we have the unique solution at the lowest regularity $(s, l)=\left(\frac{1}{2}, 0\right)$, it gains as much regularity as the initial data. To prove this, we will focus on the derivation of a priori estimates, assuming that all relevant norms are finite, which is justified by the local well-posedness in higher regularity by Proposition 4.2.

For solutions $(u, N) \in\left(X^{1 / 2} \times Y^{0}\right)([0, T))$ with $(u(0), N(0)) \in H^{s} \times H^{l}$ and $0<T \leq \infty$, we will improve the regularity up to $H^{s} \times H^{l}$ by the following steps:

(1) Improve $u$ to $s<l+1$.

(2) Improve $N$ to $l \leq 2 s-1, l \leq s+1$ and $(s, l) \neq(2,3)$ for $s<l+1$.

(3) Improve $u$ to $1<s<4 l+1, s \leq 2 l+\frac{11}{8}$ and $s \leq l+2$. 
The persistence of regularity is a general phenomenon in nonlinear wave equations, but we encounter some difficulties. One is the same as in the previous section, which is solved by Lemma 4.1. Another difficulty for $s \geq l+1$ is that the normal form can not keep the full Strichartz norm of $u$, which is why we separate (3).

5A. Regularity upgrade for $\boldsymbol{u}$ in $\boldsymbol{s}<\boldsymbol{l}+\mathbf{1}$. Let $(s, l) \in \mathbb{R}^{2}$ satisfy $(1-11)$ and $s<l+1$. Let $\left(u_{0}, N_{0}\right)$ be in $H^{s} \times H^{l}$ and let $(u, N) \in\left(X^{1 / 2} \times Y^{l}\right)([0, T))$ be a solution for some $0<T \leq 0$. If $T=\infty$, we also assume that $N$ scatters in $H_{x}^{l}$. From the estimates in Section 2, we have, for $s<l+1$,

$$
\begin{aligned}
\left\|(N u)_{L H+H H+\alpha L}\right\|_{L_{t}^{2} B_{4 / 3}^{s}+L_{t}^{1} H_{x}^{s}} & \leq C_{1}(K)\|N\|_{L_{t}^{\infty} L_{x}^{2}+L_{t}^{2} L_{x}^{4}}\|u\|_{L_{t}^{2} B_{4}^{s}}, \\
\|\Omega(N, u)\|_{L_{t}^{\infty} H_{x}^{s}} & \leq C_{0} 2^{-\theta K}\|N\|_{L_{t}^{\infty} L_{x}^{2}}\|u\|_{L_{t}^{\infty} H_{x}^{s},} \\
\|\Omega(N, u)\|_{L_{t}^{2} B_{4}^{s}} & \leq C_{0} 2^{-\theta K}\|N\|_{L_{t}^{\infty} H_{x}^{l}}\|u\|_{L_{t}^{2} B_{4}^{s},}, \\
\left\|\Omega\left(D|u|^{2}, u\right)\right\|_{L_{t}^{1} H_{x}^{s}} & \leq C_{0} 2^{-\theta K}\|u\|_{L_{t}^{\infty} H_{x}^{s}}\|u\|_{L_{t}^{2} B_{4}^{1 / 2}}^{2}, \\
\|\Omega(N, N u)\|_{L_{t}^{2} B_{4 / 3}^{s}} & \leq C_{0} 2^{-\theta K}\|N\|_{L_{t}^{\infty} H_{x}^{l}}^{2}\|u\|_{L_{t}^{2} B_{4}^{s}}
\end{aligned}
$$

for some constants $\theta(s, l)>0, C_{0}(s, l)>0$ and $C_{1}(K, s, l)>0$. Note that $C_{0} \rightarrow \infty$ as $(s, l) \rightarrow(1,0)$ in the third and the last estimates, and the small factor $2^{-\theta K}$ is lost for $s=l+1$ in the third estimate. Anyway, taking $K=K(s, l)$ large ensures smallness of the right side in the latter 4 estimates:

$$
C_{0} 2^{-\theta K}\left\{\|N\|_{L_{t}^{\infty} H_{x}^{l}}+\|u\|_{L_{t}^{2} B_{4}^{1 / 2}}^{2}+\|N\|_{L_{t}^{\infty} H_{x}^{l}}^{2}\right\} \ll 1 .
$$

After fixing such $K$, choose $\varepsilon>0$ such that $C_{1}(K) \varepsilon \ll 1$, and apply Lemma 4.1 to $N$, which yields a finite sequence $0=T_{0}<T_{1}<\cdots<T_{n+1}=T$ such that

$$
\|N\|_{\left(L_{t}^{\infty} L_{x}^{2}+L_{t}^{2} L_{x}^{4}\right)\left(T_{j}, T_{j+1}\right)}<\varepsilon .
$$

Then on each subinterval we obtain, from the above estimates,

$$
\|u\|_{X^{s}\left(T_{j}, T_{j+1}\right)} \leq C_{2}\left\|u\left(T_{j}\right)\right\|_{H^{s}}+\frac{1}{2}\|u\|_{X^{s}\left(T_{j}, T_{j+1}\right)}
$$

for some constant $C_{2}(s)>0$. Hence, if $u(0) \in H^{s}$ then, by induction on $j$, we deduce that $u \in X^{s}([0, T)$ ). If $T=\infty$, this implies the scattering of $u$ in $H^{s}$, via the argument in Section 3 .

For continuous dependence on the initial data, consider a sequence of solutions $\left(u_{n}, N_{n}\right)$ such that $\left(u_{n}(0), N_{n}(0)\right) \rightarrow(u(0), N(0))$ in $H^{s} \times H^{l}, u_{n} \rightarrow u$ in $X^{1 / 2}(I)$ and $N_{n} \rightarrow N$ in $Y^{l}(I)$ for some interval $I \subset[0, T)$. For large $n,\left(u_{n}, N_{n}\right)$ satisfies similar bounds to (5-2) and (5-3) within $I$, with slightly bigger bounds. Then the same estimates as above for $\left(u_{n}-u, N_{n}-N\right)$ yield the convergence in $\left(X^{s} \times Y^{l}\right)(I)$.

5B. Regularity upgrade for $\boldsymbol{N}$ in $\boldsymbol{s}<\boldsymbol{l}+\mathbf{1}$. Let $(s, l) \in \mathbb{R}^{2}$ satisfy (1-11) and $s<l+1$. Let $\left(u_{0}, N_{0}\right)$ be in $H^{s} \times H^{l}$ and let $(u, N) \in\left(X^{s} \times Y^{l^{\prime}}\right)([0, T))$ for some $0<T \leq \infty$ and some $l^{\prime} \in(s-1, l)$. From the 
estimates in Section 2, we have

$$
\begin{aligned}
&\left\|D\left(|u|^{2}\right)_{H H+L \alpha+\alpha L}\right\|_{L_{t}^{1} H_{x}^{l}} \leq C_{1}(K)\|u\|_{L_{t}^{2} B_{4}^{s}}^{2}, \\
&\|D \tilde{\Omega}(u, u)\|_{L_{t}^{\infty} H_{x}^{l}} \leq C_{0}\|u\|_{L_{t}^{\infty} H_{x}^{s}}^{2}, \\
&\|D \tilde{\Omega}(N u, u)\|_{L_{t}^{1} H_{x}^{l}}+\|D \tilde{\Omega}(u, N u)\|_{L_{t}^{1} H_{x}^{l}} \leq C_{0}\|N\|_{L_{t}^{\infty} H_{x}^{l}}\|u\|_{L_{t}^{2} B_{4}^{s}}^{2}
\end{aligned}
$$

for some constants $C_{0}(s, l)>0$ and $C_{1}(K, s, l)>0$, and the same for $D \tilde{\Omega}(u, N u)$. Choose $\varepsilon>0$ so small that $C_{0} \varepsilon^{2} \ll 1$. Since $u \in L_{t}^{2} B_{4}^{s}(0, T)$, there exists a finite sequence $0=T_{0}<T_{1}<\cdots<T_{n+1}=T$ such that

$$
\|u\|_{L_{t}^{2} B_{4}^{s}\left(T_{j}, T_{j+1}\right)}<\varepsilon
$$

for each $j$. Then on each subinterval we have, from the above estimates,

$$
\|N\|_{L_{t}^{\infty} H_{x}^{l}\left(T_{j}, T_{j+1}\right)} \leq C_{2}\left\|N\left(T_{j}\right)\right\|_{H^{l}}+\frac{1}{2}\|N\|_{L_{t}^{\infty} H_{x}^{l}\left(T_{j}, T_{j+1}\right)}+C_{1}(K) \varepsilon^{2}+C_{0}\|u\|_{L_{t}^{\infty} H_{x}^{s}\left(T_{j}, T_{j+1}\right)}^{2},
$$

for some constant $C_{2}(l)>0$. Hence, if $N(0) \in H^{l}$ then, by induction on $j$, we deduce that $N \in L_{t}^{\infty} H^{l}(0, T)$. If $T=\infty$, then we have the scattering of $N$ from the argument in Section 3. We also obtain the Strichartz norm of $N$ using (2-15) for the normal form. We can also upgrade continuous dependence, using the same estimates for the difference from the limit; see the previous subsection for more detail. Combining the results in this and the previous subsections yields:

Proposition 5.1. Let $(s, l) \in \mathbb{R}^{2}$ satisfy (1-11) and $s<l+1$. Let $(u, N) \in\left(X^{1 / 2} \times Y^{0}\right)(I)$ be a solution of (1-9) on an interval $I \subset \mathbb{R}$, and suppose that $\left(u\left(t_{0}\right), N\left(t_{0}\right)\right) \in H^{s}\left(\mathbb{R}^{4}\right) \times H^{l}\left(\mathbb{R}^{4}\right)$ at some $t_{0} \in I$. Then $(u, N) \in\left(X^{s} \times Y^{l}\right)(I)$ and, moreover,

$$
N \in L_{t}^{2}\left(I ; \dot{B}_{6}^{l-5 / 6} \cap \dot{B}_{6}^{-5 / 6}\right) .
$$

If $I \supset\left(t_{0}, \infty\right)$, then $(u, N)$ scatters in $H^{s} \times H^{l}$ as $t \rightarrow \infty$. If $\left(u_{n}\left(t_{0}\right), N_{n}\left(t_{0}\right)\right) \rightarrow\left(u\left(t_{0}\right), N\left(t_{0}\right)\right)$ in $H^{s} \times H^{l}$ and the corresponding sequence of solutions $\left(u_{n}, N_{n}\right) \rightarrow(u, N)$ in $\left(X^{1 / 2} \times Y^{0}\right)(J)$ on some interval $t_{0} \in J \subset I$, then the convergence holds in $\left(X^{s} \times Y^{l}\right)(J)$. The same convergence result holds for the scattering data if $I \cap J \supset\left(t_{1}, \infty\right)$ for some $t_{1}<\infty$.

5C. Regularity upgrade for $\boldsymbol{u}$ in $s \geq l+1$. Let $(s, l) \in \mathbb{R}^{2}$ satisfy $s \geq l+1$. Then $l>0$ and $s>1$. Let $\left(u_{0}, N_{0}\right) \in H^{s} \times H^{l}$ and let $(u, N) \in\left(X^{s^{\prime}} \times Y^{l}\right)([0, T))$ for some $0<T \leq \infty$ and some $s^{\prime} \in(1, s)$. In this case, the normal form estimate is not good enough to keep the full Strichartz bound of $u$. Hence we decompose

$$
\begin{aligned}
u & =u^{\prime}+\Omega(N, u), \\
\left(i \partial_{t}-\Delta\right) u^{\prime} & =(N u)_{\widetilde{L H}}+\Omega\left(\alpha D|u|^{2}, u\right)+\Omega(N, N u),
\end{aligned}
$$

where $\widetilde{L H}:=L H+H H+\alpha L$ for brevity, and look for closed estimates in

$$
\begin{aligned}
& u^{\prime} \in X^{s}, \quad u \in X^{\prime}:=L_{t}^{\infty} H_{x}^{s} \cap L_{t}^{2 /(1-\gamma)} L_{x}^{\infty}, \\
& N \in L_{t}^{\infty} H_{x}^{l} \cap L_{t}^{2 / \gamma} \mathscr{B}, \quad \mathscr{B}:=\dot{B}_{q_{1}}^{l-5 \gamma / 6} \cap \dot{B}_{q_{1}}^{-5 \gamma / 6}
\end{aligned}
$$


with $1 / q_{1}:=\frac{1}{2}-\frac{1}{3} \gamma$ for some $\gamma \in\left[0, \frac{3}{4}\right]$ satisfying

$$
\gamma+1<s, \quad 2 l+\frac{1}{2} \gamma+1 \geq s .
$$

Such $\gamma$ exists if and only if $1<s<4 l+1$ and $s \leq 2 l+\frac{11}{8}$. Also note that

$$
X^{s} \subset L_{t}^{2 /(1-\gamma)} B_{4 /(1+\gamma)}^{s} \subset L_{t}^{2 /(1-\gamma)} L_{x}^{\infty}
$$

since $\gamma+1<s$. Similarly, $L_{t}^{2 / \gamma} \mathscr{B}$ is a wave-Strichartz norm in $H^{l}$; see (5-8).

We write $(N u)_{\widetilde{L H}}=\left(N u^{\prime}\right)_{\widetilde{L H}}+(N \Omega(N, u))_{\widetilde{L H}}$. From the estimates in Section 2, we have, for $k>2$,

$$
\begin{gathered}
\left\|\left(N u^{\prime}\right) \widetilde{L H}\right\|_{L_{t}^{2} B_{4 / 3}^{s}+L_{t}^{1} H_{x}^{s}} \leq C_{1}(K)\|N\|_{L_{t}^{\infty} L_{x}^{2}+L_{t}^{2} L_{x}^{4}}\left\|u^{\prime}\right\|_{L_{t}^{2} B_{4}^{s}}, \\
\left\|\Omega\left(D|u|^{2}, u\right)\right\|_{L_{t}^{1} H_{x}^{s}} \leq C_{0} 2^{-\theta K}\|u\|_{L_{t}^{2} B_{4}^{1 / 2}}^{2}\|u\|_{L_{t}^{\infty} H_{x}^{s}}, \\
\left\|P_{>k} \Omega(N, u)\right\|_{L_{t}^{\infty} H_{x}^{s}} \leq C_{0}\left\|N_{>k-1}\right\|_{L_{t}^{\infty} H_{x}^{l}}\|u\|_{L_{t}^{\infty} H_{x}^{s}}
\end{gathered}
$$

for some constants $C_{0}(s, l)>0, \theta(s)>0$ and $C_{1}(K, s, l)>0$. We need some more estimates. Since $H^{l+2} \subset L_{x}^{\infty}$ we have, for $k>2$,

$$
\left\|P_{>k} \Omega(N, u)\right\|_{L_{t}^{2 /(1-\gamma)} L_{x}^{\infty}} \leq C_{0}\left\|N_{>k-1}\right\|_{L_{t}^{\infty} H_{x}^{l}}\|u\|_{L_{t}^{2 /(1-\gamma)} L_{x}^{\infty}}
$$

It remains to estimate $\Omega(N, N u)$ and $(N \Omega(N, u))_{\widetilde{L H}}$. If $\mathscr{B} \subset L_{x}^{4}$ then, for $k \gg\langle\log \alpha\rangle$,

$$
\begin{aligned}
&\left\|P_{>k} \Omega(N, N u)\right\|_{B_{4 / 3}^{l+2}} \lesssim\left\|N_{>k-1}\right\|_{H_{x}^{l}}\|N u\|_{L_{x}^{4}} \lesssim\left\|N_{>k-1}\right\|_{H_{x}^{l}}\|N\|\left\|_{\mathscr{B}}\right\| u \|_{L_{x}^{\infty}}, \\
&\left\|P_{>k}(N \Omega(N, u))_{\widetilde{L H}}\right\|_{B_{4 / 3}^{l+2}} \lesssim\|N\|_{\mathscr{B}}\left\|N_{>k-K-3}\right\|_{H_{x}^{l}}\|u\|_{L_{x}^{\infty}}
\end{aligned}
$$

If $\mathscr{B} \not \subset L_{x}^{4}$ but $l \geq \frac{5}{6} \gamma$, then, putting

$$
\frac{1}{q_{2}}:=\frac{1}{q_{1}}-\frac{l-\frac{5}{6} \gamma}{4}=\frac{1}{2}-\frac{\gamma}{8}-\frac{l}{4}, \quad \frac{1}{q_{3}}:=\frac{1}{2}+\frac{1}{q_{2}},
$$

we have $\mathscr{B} \subset L^{q_{2}}$ and $B_{4 / 3}^{s} \supset B_{4 / 3}^{2 l+\gamma / 2+1} \supset B_{q_{3}}^{l+2}$, and so

$$
\begin{aligned}
\left\|P_{>k} \Omega(N, N u)\right\|_{B_{4 / 3}^{s}} & \lesssim\left\|N_{>k-1}\right\|_{H_{x}^{l}}\|N u\|_{L^{q^{2}}} \lesssim\left\|N_{>k-1}\right\|_{H_{x}^{l}}\|N\|_{\Re}\|u\|_{L_{x}^{\infty}}, \\
\left\|P_{>k}(N \Omega(N, u))_{\widetilde{L H}}\right\|_{B_{4 / 3}^{s}} & \lesssim\|N\|_{\Re}\left\|N_{>k-K-3}\right\|_{H^{l}}\|u\|_{L_{x}^{\infty}} .
\end{aligned}
$$

If $l<\frac{5}{6} \gamma$ then, using

we have

$$
B_{4 / 3}^{s} \supset B_{4 / 3}^{2 l+\gamma / 2+1} \supset B_{q_{4}}^{2 l-5 \gamma / 6+2}, \quad \frac{1}{q_{4}}:=\frac{1}{2}+\frac{1}{q_{1}}=1-\frac{\gamma}{3},
$$

$$
\left\|P_{>k}(N \Omega(N, u))_{\widetilde{L H}}\right\|_{B_{4 / 3}^{s}} \lesssim\|N\|_{\mathscr{B}}\left\|\Omega(N, u)_{>k-K-2}\right\|_{H^{l+2}} \lesssim\|N\|_{\mathscr{B}}\left\|N_{>k-K-3}\right\|_{H^{l}}\|u\|_{L_{x}^{\infty}} .
$$

For the other term, putting $\sigma:=\frac{5}{6} \gamma-l>0$ and $\beta:=l /(l+\sigma) \in(0,1)$, we have the complex interpolation

$$
\left[H^{l}, B_{q_{1}}^{-\sigma}\right]_{\beta}=B_{q_{5}}^{0} \subset L^{q_{5}}, \quad\left[H^{l}, B_{q_{1}}^{-\sigma}\right]_{1-\beta}=B_{q_{6}}^{l-\sigma},
$$


where $1 / q_{5}:=\frac{1}{2}(1-\beta)+\beta / q_{1}$ and $1 / q_{6}:=1 / q_{4}-1 / q_{5}$, whereas

$$
\begin{aligned}
\left\|P_{>k} \Omega(N, N u)\right\|_{B_{4 / 3}^{s}} \lesssim\left\|P_{>k} \Omega(N, N u)\right\|_{B_{q_{4}}^{l-\sigma+2}} & \lesssim\left\|N_{>k-1}\right\|_{B_{q_{6}}^{l-\sigma}\|N u\|_{L^{q_{5}}}} \\
& \lesssim\left\|N_{>k-1}\right\|_{B_{q_{6}}^{l-\sigma}\|N\|_{B_{q_{5}}^{0}}\|u\|_{L_{x}^{\infty} .}}
\end{aligned}
$$

Hence, by the interpolation inequality,

$$
\|\Omega(N, N u)\|_{B_{4 / 3}^{s}} \lesssim\left\|N_{>k-1}\right\|_{H^{l}}^{\beta}\|N\|_{H^{l}}^{1-\beta}\|N\|_{\Re}\|u\|_{L_{x}^{\infty}} .
$$

Therefore, in any case we have some $\beta(l, \gamma) \in(0,1]$ such that

$$
\begin{aligned}
&\left\|P_{>k} \Omega(N, N u)\right\|_{L_{t}^{2} B_{4 / 3}^{s}} \leq C_{2}\left\|N_{>k-1}\right\|_{L_{t}^{\infty} H_{x}^{l}}^{\beta}\|N\|_{L_{t}^{\infty} H_{x}^{l}}^{1-\beta}\|N\|_{L_{t}^{2 / \gamma}}\|u\|_{X^{\prime}}, \\
&\left\|P_{>k}(N \Omega(N, u))_{\widetilde{L H}}\right\|_{L_{t}^{2} B_{4 / 3}^{s}} \leq C_{2}\left\|N_{>k-K-3}\right\|_{L_{t}^{\infty} H_{x}^{l}}\|N\|_{L_{t}^{2 / \gamma} \mathscr{B}}\|u\|_{X^{\prime}}
\end{aligned}
$$

for some constant $C_{2}(s, l)>0$. Choose $K \gg 1$ so large that $C_{0} 2^{-\theta K}\|u\|_{L_{t}^{2} B_{4}^{1 / 2}}^{2} \ll 1$, and then choose $\varepsilon>0$ so small and $k \gg K$ so large that

$$
\begin{array}{r}
C_{1}(K) \varepsilon+C_{0}\left\|N_{>k-1}\right\|_{L_{t}^{\infty} H_{x}^{l}} \ll 1, \\
C_{2}\left\|N_{>k-K-3}\right\|_{L_{t}^{\infty} H_{x}^{l}}^{\beta}\|N\|_{L_{t}^{\infty} H_{x}^{l}}^{1-\beta}\|N\|_{L_{t}^{2 / \gamma} \mathscr{\beta}} \ll 1 .
\end{array}
$$

Applying Lemma 4.1, we obtain a finite sequence $0=T_{0}<T_{1}<\cdots<T_{n+1}=T$ such that (5-3) holds. Then, from the above estimates, on each subinterval,

$$
\begin{aligned}
\left\|u_{>k}^{\prime}\right\|_{X^{s}\left(T_{j}, T_{j+1}\right)} & \leq C_{3}\left\|u^{\prime}\left(T_{j}\right)\right\|_{H^{s}}+\delta\left\|u^{\prime}\right\|_{X^{s}\left(T_{j}, T_{j+1}\right)}+\delta\|u\|_{X^{\prime}\left(T_{j}, T_{j+1}\right)}, \\
\left\|\Omega(N, u)_{>k}\right\|_{X^{\prime}\left(T_{j}, T_{j+1}\right)} & \leq \delta\|u\|_{X^{\prime}\left(T_{j}, T_{j+1}\right)}
\end{aligned}
$$

for some small constant $\delta>0$, while the frequencies below $k$ are bounded by $X^{1 / 2}$. Using $u=u^{\prime}+\Omega(N, u)$ and $X^{s} \subset X^{\prime}$, and adding the low frequencies, we obtain

$$
\left\|u^{\prime}\right\|_{X^{s}\left(T_{j}, T_{j+1}\right)}+\|\Omega(N, u)\|_{X^{\prime}\left(T_{j}, T_{j+1}\right)} \leq 2 C_{3}\left\|u^{\prime}\left(T_{j}\right)\right\|_{H^{s}}+2^{k(s-1 / 2)}\|u\|_{X^{1 / 2}\left(T_{j}, T_{j+1}\right)} .
$$

By induction on $j$ starting from $\left\|u^{\prime}(0)\right\|_{H^{s}}<\infty$, we thus obtain

$$
\|u\|_{X^{\prime}(0, T)} \lesssim\left\|u^{\prime}\right\|_{X^{s}(0, T)}+\|\Omega(N, u)\|_{X^{\prime}(0, T)}<\infty .
$$

If $T=\infty$, then $u$ is scattering, by the argument in Section 3. Thus we have obtained:

Proposition 5.2. Let $(s, l) \in \mathbb{R}^{2}$ satisfy (1-11) and $s \geq l+1$. Let $(u, N) \in\left(X^{1 / 2} \times Y^{0}\right)(I)$ be a solution of (1-9) on an interval $I \subset \mathbb{R}$, and suppose that $\left(u\left(t_{0}\right), N\left(t_{0}\right)\right) \in H^{s}\left(\mathbb{R}^{4}\right) \times H^{l}\left(\mathbb{R}^{4}\right)$ at some $t_{0} \in I$. Then we have $u-\Omega(N, u) \in X^{s}(I)$, as well as (5-8), and, for all $\gamma \in\left[0, \frac{3}{4}\right]$ satisfying (5-11),

$$
u \in C\left(I ; H_{x}^{s}\right) \cap L_{t}^{\infty}\left(I ; H_{x}^{s}\right) \cap L^{2 /(1-\gamma)}\left(I ; L_{x}^{\infty}\right) .
$$

We also have scattering and continuous dependence similar to Proposition 5.1, but in the space (5-28).

It is easy to replace $L_{x}^{\infty}$ with $B_{4 /(1+\gamma)}^{s}+H^{l+2}$ using $u^{\prime} \in X^{s}$ and (5-14). 
5D. Lipschitz continuity of the solution map. Here we consider local Lipschitz continuity of the flow map. In the above arguments, the Lipschitz dependence is lost only when we seek time intervals with smallness, typically by Lemma 4.1. If $\left(u_{0}, N_{0}\right) \in H^{s} \times H^{l}$ with $s>\frac{1}{2}$ and $l>0$, however, it is easy to see that (4-5) holds locally uniformly with respect to the initial data, because we can first dispose of the high frequencies using the higher regularity, and then the remaining low frequencies by Sobolev in $x$ and Hölder in $t$.

Similarly, the regularity upgrading argument in Section 5A works uniformly if $l>0$ and $T<\infty$, because of (5-3), and so does the argument in Section 5B for $s>\frac{1}{2}, l<\min (2 s-1, s+1)$, and $T<\infty$, because of (5-6), as well as that in Section 5C for $l>0, s<\min \left(2 l+\frac{11}{8}, l+2\right)$, and $T<\infty$, because of (5-24) and (5-3).

Thus we obtain Lipschitz continuity of the flow map, locally both in time and in the initial data, for all the exponents $(s, l)$ in the range and off the boundary. Since we need to decrease $l$ for the uniform control in (5-24), $\gamma$ in (5-10) cannot be on the boundary, namely $2 l+\frac{1}{2} \gamma+1>s$, for the local Lipschitz estimate.

The Lipschitz continuity global in time and for the scattering is more tricky, because the $L_{t}^{2} L_{x}^{4}$ norm in Lemma 4.1 is not bounded by the Strichartz estimate for the wave equation. For small data, we can obtain Lipschitz estimates directly from the contraction mapping argument, but then the smallness on $H^{1 / 2} \times L^{2}$ depends on $(s, l)$, which tends to 0 as $(s, l)$ approaches $s=4 l+1,(2,3)$ or $(\infty, \infty)$. The regularity upgrading for $N$ in Section 5B works well for $T=\infty$, because in (5-6) the number of subintervals can be uniformly bounded for each $\varepsilon>0$, provided that $\|u\|_{L_{t}^{2} B_{4}^{s}}$ is uniformly bounded. This yields a smallness condition in the form

$$
\left\|\left(u_{0}, N_{0}\right)\right\|_{H^{1 / 2} \times L^{2}} \leq \varepsilon_{2}(s, l),
$$

where $\varepsilon_{2}(s, l)>0$ is nondecreasing in $l$ for global Lipschitz continuity in $H^{s} \times H^{l}$.

Remark 5.3. Strictly speaking, we need to prove that the solution to (2-5) obtained above is also a solution of (1-9) before the normal form. The easiest way is to use [Ginibre et al. 1997] for existence of solutions for smooth approximating initial data, taking the limit by the continuous dependence proved above. To be self-contained, however, we can directly show that smooth solutions of (2-5) solve (1-9). In fact, if $(u, N) \in\left(X^{s} \times Y^{s}\right)(I)$ with $s \gg 1$ is a solution of (2-5) on some interval $I$, then, by definition of $\Omega$ and $\tilde{\Omega},(2-5)$ reads

$$
\begin{aligned}
e q_{u} & :=\left(i \partial_{t}+D^{2}\right) u-N u=-\Omega\left(e q_{N}, u\right)-\Omega\left(N, e q_{u}\right), \\
e q_{N} & :=\left(i \partial_{t}+\alpha D\right) N-\alpha D|u|^{2}=-D \tilde{\Omega}\left(e q_{u}, u\right)-D \tilde{\Omega}\left(u, e q_{u}\right) .
\end{aligned}
$$

Since $e q_{u}, e q_{N} \in C\left(I ; H^{s-2}\right)$ and $\Omega, D \tilde{\Omega}:\left(H^{s-2}\right)^{2} \rightarrow H^{s-2}$ has a small factor due to $K$, we deduce that $e q_{u}=0=e q_{N}$ on $I$ if $K$ is large enough.

\section{Small data scattering in the energy space}

For $(s, l)=(1,0)$, the failure of Strichartz bound on the normal form $\Omega(N, u)$ cannot be compensated by regularity of $N$, and so there seems no way to close the estimates as above for $(s, l)=(1,0)$. Instead, we invoke the conservation laws with the weak compactness argument. This type of argument usually yields 
a weak result, typically without uniqueness. We can however obtain the strong well-posedness for small data as in Theorem 1.1, thanks to that both in the larger space $(s, 0)$ with $s<1$, and in the smaller space $(1, l)$ with $l>0$.

Assume that $\left(u_{0}, N_{0}\right) \in H^{1} \times L^{2}$. By Proposition 3.1 there is $\varepsilon_{0}:=\varepsilon_{1}\left(\frac{1}{2}, 0\right) \ll 1$ such that, if $\left\|\left(u_{0}, N_{0}\right)\right\|_{H^{1 / 2} \times L^{2}} \leq \varepsilon_{0}$, then there is a unique global solution $(u, N)$ in $X^{1 / 2} \times Y^{0}$, satisfying

$$
\|(u, N)\|_{X^{1 / 2} \times Y^{0}} \leq C \varepsilon_{0} \ll 1 .
$$

Proposition 5.1 implies that $(u, N) \in X^{s} \times Y^{0}$ for all $s \in\left[\frac{1}{2}, 1\right)$.

Fix a sequence $\left\{\left(u_{0, n}, N_{0, n}\right)\right\} \subset \mathscr{S}\left(\mathbb{R}^{4}\right)$ such that

$$
\left(u_{0, n}, N_{0, n}\right) \rightarrow\left(u_{0}, N_{0}\right) \quad \text { in } H^{1} \times L^{2} \quad \text { and } \quad\left\|\left(u_{0, n}, N_{0, n}\right)\right\|_{H^{1 / 2} \times L^{2}} \leq \varepsilon_{0} .
$$

By Proposition 3.1, for each $n$, there is a unique global solution $\left(u_{n}, N_{n}\right)$ satisfying (6-1) and, for all $\frac{1}{2} \leq s<1$,

$$
\sup _{n}\left\|\left(u_{n}, N_{n}\right)\right\|_{X^{s} \times Y^{0}}<\infty .
$$

Now we claim a uniform bound at the energy level:

$$
\sup _{n, t}\left\|\left(u_{n}(t), N_{n}(t)\right)\right\|_{H^{1} \times L^{2}}<\infty
$$

By Proposition 5.1, we have $\left(u_{n}, N_{n}\right) \in X^{8} \times Y^{9}$ for all $n$, by which we can justify the conservation law $E_{Z}\left(u_{n}(t), N_{n}(t)\right)=E_{Z}\left(u_{0, n}, N_{0, n}\right)$. Using (6-1) for $N_{n}$ together with the Sobolev inequality $\|u\|_{L_{x}^{4}} \lesssim\|\nabla u\|_{L_{x}^{2}}$ yields

$$
E_{Z}\left(u_{n}, N_{n}\right)=\left(1-O\left(\varepsilon_{0}\right)\right)\left\|\nabla u_{n}\right\|_{2}^{2}+\frac{1}{2}\left\|N_{n}\right\|_{2}^{2},
$$

which, combined with the lower regularity bound (6-2), implies (6-3).

Next we prove convergence $u_{n}(t) \rightarrow u(t)$ in $H_{x}^{1}$ as $n \rightarrow \infty$, locally uniformly in $\mathbb{R}$. Take any convergent sequence $t_{n} \rightarrow t_{\infty}$. From Propositions 3.1 and 5.1, we know that $u_{n}\left(t_{n}\right) \rightarrow u\left(t_{\infty}\right)$ in $H_{x}^{s}$ for $s<1$, and $N_{n}\left(t_{n}\right) \rightarrow N\left(t_{\infty}\right)$ in $L_{x}^{2}$. From (6-3), we have $\left\{u_{n}\left(t_{n}\right)\right\}_{n}$ is bounded in $H_{x}^{1} \subset L_{x}^{4}$, thus we get $u\left(t_{\infty}\right) \in H^{1}$, $u_{n}\left(t_{n}\right) \rightarrow u\left(t_{\infty}\right)$ weakly in $H_{x}^{1}$, and $\left|u_{n}\left(t_{n}\right)\right|^{2} \rightarrow\left|u\left(t_{\infty}\right)\right|^{2}$ weakly in $L_{x}^{2}$. Since $N_{n}\left(t_{n}\right) \rightarrow N\left(t_{\infty}\right)$ strongly in $L_{x}^{2}$, we have $\int N_{n}\left(t_{n}\right)\left|u_{n}\left(t_{n}\right)\right|^{2} d x \rightarrow \int N\left(t_{\infty}\right)\left|u\left(t_{\infty}\right)\right|^{2} d x$, and so

$$
E_{Z}\left(u\left(t_{\infty}\right), N\left(t_{\infty}\right)\right) \leq \liminf _{n \rightarrow \infty} E_{Z}\left(u_{n}\left(t_{n}\right), N_{n}\left(t_{n}\right)\right)=\liminf _{n \rightarrow \infty} E_{Z}\left(u_{0, n}, N_{0, n}\right)=E_{Z}\left(u_{0}, N_{0}\right)
$$

By the time reversibility we get $E_{Z}\left(u\left(t_{\infty}\right), N\left(t_{\infty}\right)\right)=E_{Z}\left(u_{0}, N_{0}\right)$. Indeed, if there is a $t_{0} \in \mathbb{R}$ such that $E_{Z}\left(u\left(t_{0}\right), N\left(t_{0}\right)\right)<E_{Z}\left(u_{0}, N_{0}\right)$, then we solve the Zakharov system with initial data $\left(u\left(t_{0}\right), N\left(t_{0}\right)\right)$ at $t=t_{0}$. By the uniqueness we get a contradiction. Then the equality in (6-5) implies $\left\|\nabla u_{n}\left(t_{n}\right)\right\|_{L^{2}} \rightarrow\left\|\nabla u\left(t_{\infty}\right)\right\|_{L^{2}}$, from which we conclude that $u_{n}\left(t_{n}\right) \rightarrow u\left(t_{\infty}\right)$ strongly in $H_{x}^{1}$, and so the locally uniform convergence $u_{n} \rightarrow u$ in $C\left(\mathbb{R} ; H_{x}^{1}\right)$. Thus we obtain the unique global solution $(u, N) \in\left(C \cap L^{\infty}\right)\left(\mathbb{R} ; H^{1} \times L^{2}\right)$. Note that the smoothness of the approximate solutions $\left(u_{n}, N_{n}\right)$ was used only to ensure the unique existence and the conservation law. Now that we have them for the solutions in the energy space, we can apply the 
above argument to a sequence of initial data in $H^{1} \times L^{2}$, which implies continuous dependence of the initial data, locally uniformly in time.

By Propositions 3.1 and 5.1, $(u, N)$ scatters to some $\left(u^{+}, N^{+}\right)$in $H^{s} \times L^{2}$ for all $s<1$. Since $u(t) \in L^{\infty}\left(\mathbb{R} ; H_{x}^{1}\right)$, we have $S(-t) u(t) \rightarrow u^{+}$weakly in $H^{1}$ as $t \rightarrow+\infty$. Since $|u(t)|^{2}$ is bounded in $\left(H_{x}^{1}\right)^{2} \subset B_{4 / 3}^{1}$, while $N(t)$ is vanishing in $B_{4}^{-1}$ as $t \rightarrow \infty$ due to the scattering in $L_{x}^{2} \subset B_{4}^{-1}$, we have

$$
\int N(t)|u(t)|^{2} d x \rightarrow 0 \quad(t \rightarrow+\infty)
$$

and so

$$
\begin{aligned}
\left\|\nabla u^{+}\right\|_{2}^{2}+\frac{1}{2}\left\|N^{+}\right\|_{2}^{2} & \leq \liminf _{t \rightarrow+\infty}\|\nabla S(-t) u(t)\|_{2}^{2}+\frac{1}{2}\left\|W_{\alpha}(-t) N(t)\right\|_{2}^{2} \\
& =\liminf _{t \rightarrow+\infty}\|\nabla u(t)\|_{2}^{2}+\frac{1}{2}\|N(t)\|_{2}^{2} \\
& =\liminf _{t \rightarrow+\infty} E_{Z}(u(t), N(t))=E_{Z}\left(u_{0}, N_{0}\right) .
\end{aligned}
$$

To prove the equality above, we consider the final state problem. Following the argument in the first step, above, we fix a sequence $\left\{\left(u_{n}^{+}, N_{n}^{+}\right)\right\} \subset \mathscr{Y}\left(\mathbb{R}^{4}\right)$ such that $\left(u_{n}^{+}, N_{n}^{+}\right) \rightarrow\left(u^{+}, N^{+}\right)$in $H^{1} \times L^{2}$. Then, by Proposition 3.1, we have a sequence of solutions $\left(\tilde{u}_{n}, \tilde{N}_{n}\right) \in X^{1 / 2} \times Y^{0}$ scattering to $\left(u_{n}^{+}, N_{n}^{+}\right)$as $t \rightarrow \infty$, which converges to $(u, N)$ in $X^{1 / 2} \times Y^{0}$ as $n \rightarrow \infty$. The regularity is upgraded to $X^{s} \times Y^{l}$ for all $(s, l)$ in Proposition 5.1. As in the first step, we have $\sup _{n, t}\left\|\left(\tilde{u}_{n}, \tilde{N}_{n}\right)\right\|_{H^{1} \times L^{2}}<\infty$, hence $u_{n}(t) \rightarrow u(t)$ weakly in $H_{x}^{1}$. Thus, by (6-6),

$$
E_{Z}(u(t), N(t)) \leq \liminf _{n \rightarrow \infty} E_{Z}\left(\tilde{u}_{n}(t), \tilde{N}_{n}(t)\right)=\liminf _{n \rightarrow \infty}\left\|\nabla u_{n}^{+}\right\|_{2}^{2}+\frac{1}{2}\left\|N_{n}^{+}\right\|_{2}^{2}=\left\|\nabla u^{+}\right\|_{2}^{2}+\frac{1}{2}\left\|N^{+}\right\|_{2}^{2} .
$$

Hence, we get

$$
\lim _{t \rightarrow+\infty} E_{Z}(u(t), N(t))=\left\|\nabla u^{+}\right\|_{2}^{2}+\frac{1}{2}\left\|N^{+}\right\|_{2}^{2}
$$

and so $S(-t) u(t) \rightarrow u^{+}$strongly in $H_{x}^{1}$, namely the scattering in $H_{x}^{1}$.

To show the continuity of the solution map in $L_{t}^{\infty}\left(\mathbb{R} ; H_{x}^{1}\right)$, it remains to prove $u_{n}\left(t_{n}\right)-u\left(t_{n}\right) \rightarrow 0$ in $H_{x}^{1}$, in the case $t_{n} \rightarrow \infty$, for a sequence of solutions $\left(u_{n}, N_{n}\right)$ in the energy space such that

$$
\left(u_{n}(0), N_{n}(0)\right) \rightarrow(u(0), N(0)) \quad \text { in } H^{1} \times L^{2} .
$$

Since $S(-t) u(t) \rightarrow u^{+}$in $H_{x}^{1}$, this is equivalent to showing $S\left(-t_{n}\right) u_{n}\left(t_{n}\right) \rightarrow u^{+}$in $H_{x}^{1}$. We already know the $H_{x}^{s}$ convergence for $s<1$ as well as the weak convergence in $H_{x}^{1}$. Then the strong convergence is equivalent to $\left\|u_{n}\left(t_{n}\right)\right\|_{H_{x}^{1}} \rightarrow\left\|u^{+}\right\|_{H^{1}}$. Since

$$
\left\|N_{n}\left(t_{n}\right)\right\|_{B_{4}^{-1}} \leq\left\|N_{n}-N\right\|_{L_{t}^{\infty} L_{x}^{2}}+\left\|N\left(t_{n}\right)\right\|_{B_{4}^{-1}} \rightarrow 0
$$

we have $\int N_{n}\left(t_{n}\right)\left|u_{n}\left(t_{n}\right)\right|^{2} d x \rightarrow 0$, and so, as $n \rightarrow \infty$,

$$
\begin{aligned}
\left\|\nabla u_{n}\left(t_{n}\right)\right\|_{2}^{2}+\frac{1}{2}\left\|N_{n}\left(t_{n}\right)\right\|_{2}^{2}=E_{Z}\left(u_{n}\left(t_{n}\right), N_{n}\left(t_{n}\right)\right)+o(1) & =E_{Z}\left(u_{n}(0), N_{n}(0)\right)+o(1) \\
& =E_{Z}(u(0), N(0))+o(1) \\
& =\left\|\nabla u^{+}\right\|_{2}^{2}+\frac{1}{2}\left\|N^{+}\right\|_{2}^{2}+o(1) .
\end{aligned}
$$


Since $\left\|u_{n}\left(t_{n}\right)\right\|_{2} \rightarrow\left\|u^{+}\right\|_{2}$ and $\left\|N_{n}\left(t_{n}\right)\right\|_{2} \rightarrow\left\|N^{+}\right\|_{2}$, the above implies the strong convergence of $S\left(-t_{n}\right) u_{n}\left(t_{n}\right)$ in $H_{x}^{1}$, and thus $u_{n} \rightarrow u$ in $L_{t}^{\infty}\left(\mathbb{R} ; H_{x}^{1}\right)$. This completes the proof of Theorem 1.1 in the case $(s, l)=(1,0)$.

\section{Ill-posedness at $(s, l)=(2,3)$}

In this section, we prove Theorem 1.3. The main point is that the multilinear estimates fail only for the boundary quadratic term coming from the initial data. Exploiting the dispersive smoothing, we can prove that the other terms are more regular if the initial data is localized in space.

Proof of Theorem 1.3. First of all, for any initial data $\left(u_{0}, N_{0}\right) \in H^{2} \times H^{3}$, we have a unique local solution for $(s, l)$ in (1-11) satisfying $s \leq 2$ and $l \leq 3$, say $(u, N) \in\left(X^{2} \times Y^{2}\right)([0, T])$, by Propositions 4.2 and 5.1. In the Duhamel formula (2-4), the first term on the right is obviously in $C\left(\mathbb{R} ; H^{3}\right)$. The integral terms are regular thanks to the high regularity. Indeed,

$$
\begin{aligned}
\left\|D|u|_{H H+\alpha L+L \alpha}^{2}\right\|_{L_{t}^{1} H_{x}^{3}} & \lesssim\|u\|_{L_{t}^{2} B_{4}^{2}}^{2}, \\
\|D \tilde{\Omega}(N u, u)\|_{L_{t}^{1} H_{x}^{3}} & \lesssim\|N\|_{L_{t}^{\infty} H_{x}^{2}}\|u\|_{L_{t}^{2} B_{4}^{2}}^{2},
\end{aligned}
$$

and the same for $D \tilde{\Omega}(u, N u)$. To bound $D \tilde{\Omega}(u, u)$ in $H_{x}^{3}$, we use local smoothing for $u$, assuming that

$$
u_{0} \in W^{2,1}\left(\mathbb{R}^{4}\right)=\left\{f \mid \partial^{\alpha} f \in L^{1}\left(\mathbb{R}^{4}\right) \text { for }|\alpha| \leq 2\right\}
$$

Then $S(t) u_{0} \in C\left((0, \infty) ; B_{p}^{2}\right)$ for all $p>2$ by the dispersive $L_{x}^{p}$ decay estimate for $S(t)$. Moreover, in the Duhamel formula (2-3) of $u$, the terms except for $(N u) \widetilde{L H}$ easily gain better regularity by

$$
\begin{aligned}
\|\Omega(N, u)\|_{H_{x}^{3}} & \lesssim\|N\|_{H_{x}^{2}}\|u\|_{H_{x}^{2}}, \\
\left\|\Omega\left(D|u|^{2}, u\right)\right\|_{L_{t}^{1} H_{x}^{3}} & \lesssim\|u\|_{L_{t}^{\infty} H_{x}^{2}}\|u\|_{L_{t}^{2} B_{4}^{2}}^{2}, \\
\|\Omega(N, N u)\|_{L_{t}^{2} B_{4 / 3}^{4}} & \lesssim\|N\|_{L_{t}^{\infty} H_{x}^{2}}^{2}\|u\|_{L_{t}^{2} B_{4}^{2}}
\end{aligned}
$$

The remaining term is bounded in $C\left([0, T] ; B_{3}^{2}\right)$ by

$$
\left\|\int_{0}^{t} S(t-s)(N u) \widetilde{L H} d s\right\|_{B_{3}^{2}} \lesssim \int_{0}^{t}|t-s|^{2 / 3}\left\|(N u)_{\widetilde{L H}}\right\|_{B_{3 / 2}^{2}} d s \lesssim \int_{0}^{t}|t-s|^{2 / 3}\|N(s)\|_{L_{x}^{6}}\|u(s)\|_{H_{x}^{2}} d s .
$$

Gathering the above estimates, we obtain $u \in C\left((0, T] ; H^{2} \cap B_{3}^{2}\right)$. Since $B_{3}^{2} \subset L^{\infty}$,

$$
\|D \tilde{\Omega}(u, u)\|_{H^{3}} \lesssim\|u\|_{H^{2}}\|u\|_{B_{3}^{2}}
$$

and, plugging this into the above estimates for $N$, we deduce that

$$
N-W_{\alpha}(t) D \tilde{\Omega}\left(u_{0}, u_{0}\right) \in C\left((0, T] ; H_{x}^{3}\right)
$$

if $u_{0} \in H^{2} \cap W^{2,1}\left(\mathbb{R}^{4}\right)$. Hence, it suffices to find such a $u_{0}$ such that $D \tilde{\Omega}\left(u_{0}, u_{0}\right) \notin H_{x}^{3}$. This is constructed in Lemma 7.1. Then $N(t) \notin H_{x}^{3}$ for all $0<t<T$, namely the instant exit or the latter part of the theorem. 
Thanks to the high regularity, it is easy to translate it to nonexistence. Indeed, if $(u, N)$ is in $L^{2}\left((0, T) ; H^{1} \times H^{3}\right)$ then, from the equation without the normal form,

$$
N u \in L_{t}^{1} H_{x}^{1} \Longrightarrow u \in C_{t} H_{x}^{1} \cap L_{t}^{2} B_{4}^{1} \Longrightarrow D|u|^{2} \in L_{t}^{1} L_{x}^{2} \Longrightarrow N \in C_{t} L_{x}^{2} .
$$

In particular, $(u, N)$ belongs to the uniqueness class at $(s, l)=\left(\frac{1}{2}, 0\right)$. Hence it should be identical with the exiting solution obtained above, satisfying $N(t) \notin H^{3}$ for all $t \neq 0$, contradicting $N \in L_{t}^{2}\left((0, T) ; H_{x}^{3}\right)$.

It remains to prove the failure of the bilinear estimate:

Lemma 7.1. There is a radial $u \in\left(H^{2} \cap W^{2,1}\right)\left(\mathbb{R}^{4}\right)$ satisfying $D \tilde{\Omega}(u, u) \notin H^{3}\left(\mathbb{R}^{4}\right)$.

This failure of the bilinear estimate comes from that $H^{2}\left(\mathbb{R}^{4}\right)$ is not an algebra, but we should be careful about cancellation in the nonlinearity. In fact, the proof below implies that $D \tilde{\Omega}(u, u)$ is bounded in $H^{3}$ for real-valued or purely imaginary $u \in H^{2}$.

Proof. Modulo a bounded operator, the symbol of $D \tilde{\Omega}$ can be approximated by

$$
\frac{\alpha|\xi|}{|\xi-\eta|^{2}-|\eta|^{2} \mp \alpha|\xi|}=\frac{\alpha}{|\xi|}+\frac{\alpha(2 \xi \cdot \eta \pm \alpha|\xi|)}{|\xi|\left(|\xi-\eta|^{2}-|\eta|^{2} \mp \alpha|\xi|\right)}
$$

in the $X L$ frequency, while, in the $L X$ frequency,

$$
\frac{\alpha|\xi|}{|\xi-\eta|^{2}-|\eta|^{2} \mp \alpha|\xi|}=\frac{-\alpha}{|\xi|}+\frac{\alpha(2 \xi \cdot(\xi-\eta) \pm \alpha|\xi|)}{|\xi|\left(|\xi-\eta|^{2}-|\eta|^{2} \mp \alpha|\xi|\right)}
$$

where the second terms are $O\left(|\xi|^{-2}\langle\right.$ Low $\left.\rangle\right)$ for $|\xi| \gg 1$, and so bounded $H^{2} \times H^{2} \rightarrow H^{3}$ for high frequency. Hence, it suffices to construct $u \in H^{2} \cap W^{1,2}$ such that $\operatorname{supp} \hat{u}(\xi)=0$ for $|\xi| \lesssim 1$ and

$$
(u \bar{u})_{H L}-(u \bar{u})_{L H} \notin H^{2}\left(\mathbb{R}^{4}\right) .
$$

Indeed, this is necessary and sufficient for $D \tilde{\Omega}(u, u) \notin H^{3}$ under the condition of supp $\hat{u}$. Note that the left side is simply zero if $u\left(\mathbb{R}^{4}\right) \subset \mathbb{R}$ or $i u\left(\mathbb{R}^{4}\right) \subset \mathbb{R}$. The remaining is the antisymmetric part, which can be expanded by putting $u=v+i w$ :

$$
(u \bar{u})_{H L}-(u \bar{u})_{L H}=2 i\left[(w v)_{H L}-(w v)_{L H}\right] .
$$

Now it is easy to avoid the cancellation considering the forms

$$
v=\sum_{j>J} a_{j} \varphi_{j}, \quad w=\sum_{j>J} b_{j} \varphi_{j}, \quad \varphi_{j}(x)=\varphi\left(2^{j} x\right),
$$

where $J \gg \log \alpha,\{a\},\{b\} \subset[0, \infty)$, and $\varphi \in \mathscr{Y}\left(\mathbb{R}^{4} ; \mathbb{R}\right)$ is a nonzero real-valued radial function satisfying

$$
0 \leq \hat{\varphi} \leq 1, \quad \operatorname{supp} \hat{\varphi} \subset\{|| \xi|-1| \ll 1\} .
$$

Put $c:=\varphi(0)>0$. Inserting the above ansatz expands the bilinear form

$$
(v w)_{H L}-(v w)_{L H}=\sum_{j>J} \sum_{k>J}^{j-K}\left(a_{j} b_{k}-a_{k} b_{j}\right) \varphi_{j} \varphi_{k} .
$$


Since $\mathscr{F}\left(\varphi_{j} \varphi_{k}\right)$ is supported around $|\xi|=2^{j}$,

$$
\|(7-14)\|_{H^{2}}^{2} \sim \sum_{j>J}\left\|2^{2 j} \sum_{k \leq j-K}\left(a_{j} b_{k}-a_{k} b_{j}\right) \varphi_{j} \varphi_{k}\right\|_{2}^{2} .
$$

Imposing a support condition on $\{a\}$ and $\{b\}$,

$$
\operatorname{supp} a \cap \operatorname{supp} b=\varnothing,
$$

we can decouple the above as

$$
\|(7-14)\|_{H^{2}}^{2} \sim \sum_{j>J}\left\|2^{2 j} \sum_{k \leq j-K} a_{j} b_{k} \varphi_{j} \varphi_{k}\right\|_{2}^{2}+\sum_{j>J}\left\|2^{2 j} \sum_{k \leq j-K} b_{j} a_{k} \varphi_{j} \varphi_{k}\right\|_{2}^{2} .
$$

By rescaling $x \mapsto 2^{-j} x$ and using $\varphi\left(2^{k-j} x\right)=c+O\left(\left|2^{k-j} x\right|\right)$, the $L_{x}^{2}$ norm is approximated by

$$
\begin{aligned}
\left\|2^{2 j} \sum_{k \leq j-K} a_{j} b_{k} \varphi_{j} \varphi_{k}\right\|_{L_{x}^{2}} & =\left\|a_{j} \varphi(x) \sum_{k \leq j-K} b_{k} \varphi\left(2^{k-j} x\right)\right\|_{L_{x}^{2}} \\
& \geq c\left|a_{j}\right|\|\varphi\|_{L_{x}^{2}} \sum_{k \leq j-K} b_{k}-C\left\|a_{j} x \varphi(x)\right\|_{L_{x}^{2}} \sum_{k \leq j-K} b_{k} 2^{k-j} .
\end{aligned}
$$

Fix $\theta \in\left(\frac{1}{2}, \frac{3}{4}\right)$ and let

$$
a_{j}=\left\{\begin{array}{ll}
j^{-\theta} & \text { if } J<j \text { is even, } \\
0 & \text { otherwise }
\end{array} \quad b_{j}= \begin{cases}j^{-\theta} & \text { if } J<j \text { is odd } \\
0 & \text { otherwise }\end{cases}\right.
$$

Then, for $j>K+J$,

$$
\sum_{k \leq j-K} b_{k} \sim(j-K)^{1-\theta}, \quad \sum_{k \leq j-K} b_{k} 2^{k-j} \lesssim 2^{-K}
$$

and so

$$
\|(7-14)\|_{H^{2}} \gtrsim\left\|j^{-\theta}(j-K)^{1-\theta}\right\|_{\ell^{2}(j>J+K)}-C 2^{-K}\left\|j^{-\theta}\right\|_{\ell^{2}(j>J)}=\infty,
$$

since $-\theta<-\frac{1}{2}<1-2 \theta$. Also, we have

$$
\|u\|_{H^{2}} \lesssim\left\|j^{-\theta}\right\|_{\ell^{2}(j>J)}<\infty, \quad\|u\|_{W^{2,1}} \lesssim\left\|2^{-2 j} j^{-\theta}\right\|_{\ell^{1}(j>J)}<\infty .
$$

Thus we have obtained an example $u \in H^{2} \cap W^{2,1}$, as desired.

\section{Acknowledgments}

Guo is supported in part by NNSF of China (No. 11371037), Beijing Higher Education Young Elite Teacher Project (No. YETP0002), and Fok Ying Tong education foundation (No. 141003).

Herr was supported by the German Research Foundation, CRC 701.

Part of this research was carried out while the authors participated in the program "Harmonic Analysis and Partial Differential Equations" at the Hausdorff Research Institute for Mathematics in Bonn. 


\section{References}

[Bejenaru and Herr 2011] I. Bejenaru and S. Herr, "Convolutions of singular measures and applications to the Zakharov system", J. Funct. Anal. 261:2 (2011), 478-506. MR 2012g:42042 Zbl 1228.42027

[Bejenaru et al. 2009] I. Bejenaru, S. Herr, J. Holmer, and D. Tataru, "On the 2D Zakharov system with $L^{2}$-Schrödinger data", Nonlinearity 22:5 (2009), 1063-1089. MR 2010f:35383 Zbl 1173.35651

[Bourgain and Colliander 1996] J. Bourgain and J. Colliander, "On wellposedness of the Zakharov system", Int. Math. Res. Not. 1996: 11 (1996), 515-546. MR 97h:35206 Zbl 0909.35125

[Dodson 2014] B. Dodson, "Global well-posedness and scattering for the focusing, energy-critical nonlinear Schrödinger problem in dimension $d=4$ for initial data below a ground state threshold", preprint, 2014. arXiv 1409.1950

[Ginibre and Velo 2006] J. Ginibre and G. Velo, "Scattering theory for the Zakharov system", Hokkaido Math. J. 35:4 (2006), 865-892. MR 2007k:35347 Zbl 1123.35068

[Ginibre et al. 1997] J. Ginibre, Y. Tsutsumi, and G. Velo, "On the Cauchy problem for the Zakharov system”, J. Funct. Anal. 151:2 (1997), 384-436. MR 2000c:35220 Zbl 0894.35108

[Glangetas and Merle 1994a] L. Glangetas and F. Merle, "Existence of self-similar blow-up solutions for Zakharov equation in dimension two, I", Comm. Math. Phys. 160:1 (1994), 173-215. MR 95e:35195 Zbl 0808.35137

[Glangetas and Merle 1994b] L. Glangetas and F. Merle, "Concentration properties of blow-up solutions and instability results for Zakharov equation in dimension two, II", Comm. Math. Phys. 160:2 (1994), 349-389. MR 95e:35196 Zbl 0808.35138

[Guo 2014] Z. Guo, "Sharp spherically averaged Strichartz estimates for the Schrödinger equation", preprint, 2014. arXiv 1406. 2525

[Guo and Nakanishi 2014] Z. Guo and K. Nakanishi, "Small energy scattering for the Zakharov system with radial symmetry", Int. Math. Res. Not. 2014:9 (2014), 2327-2342. MR 3207369 Zbl 1303.35089

[Guo and Wang 2014] Z. Guo and Y. Wang, "Improved Strichartz estimates for a class of dispersive equations in the radial case and their applications to nonlinear Schrödinger and wave equations", J. Anal. Math. 124 (2014), 1-38. MR 3286047 Zbl 1308.35271

[Guo et al. 2013] Z. Guo, K. Nakanishi, and S. Wang, "Global dynamics below the ground state energy for the Zakharov system in the 3D radial case", Adv. Math. 238 (2013), 412-441. MR 3033638 Zbl 1284.35369

[Guo et al. 2014a] Z. Guo, S. Lee, K. Nakanishi, and C. Wang, "Generalized Strichartz estimates and scattering for 3D Zakharov system”, Comm. Math. Phys. 331:1 (2014), 239-259. MR 3232001 Zbl 1302.35336

[Guo et al. 2014b] Z. Guo, K. Nakanishi, and S. Wang, "Global dynamics below the ground state energy for the KleinGordon-Zakharov system in the 3D radial case", Comm. Partial Differential Equations 39:6 (2014), 1158-1184. MR 3200092 Zbl 1296.35191

[Guo et al. 2014c] Z. Guo, K. Nakanishi, and S. Wang, "Small energy scattering for the Klein-Gordon-Zakharov system with radial symmetry", Math. Res. Lett. 21:4 (2014), 733-755. MR 3275645 Zbl 1304.35691

[Hani et al. 2013] Z. Hani, F. Pusateri, and J. Shatah, "Scattering for the Zakharov system in 3 dimensions", Comm. Math. Phys. 322:3 (2013), 731-753. MR 3079330 Zbl 1307.35278

[Kato and Tsugawa $\geq 2015$ ] I. Kato and K. Tsugawa, "Scattering and well-posedness for the zakharov system at a critical space in four and more spatial dimensions", in preparation.

[Keel and Tao 1998] M. Keel and T. Tao, “Endpoint Strichartz estimates”, Amer. J. Math. 120 (1998), 955-980. MR 2000d:35018 Zbl 0922.35028

[Kenig and Merle 2006] C. E. Kenig and F. Merle, "Global well-posedness, scattering and blow-up for the energy-critical, focusing, non-linear Schrödinger equation in the radial case", Invent. Math. 166:3 (2006), 645-675. MR 2007g:35232 Zbl 1115.35125

[Killip and Visan 2010] R. Killip and M. Visan, "The focusing energy-critical nonlinear Schrödinger equation in dimensions five and higher", Amer. J. Math. 132 (2010), 361-424. MR 2011e:35357 Zbl 1208.35138

[Kishimoto 2013] N. Kishimoto, "Local well-posedness for the Zakharov system on the multidimensional torus", J. Anal. Math. 119 (2013), 213-253. MR 3043152 Zbl 1310.35218 
[Masmoudi and Nakanishi 2008] N. Masmoudi and K. Nakanishi, "Energy convergence for singular limits of Zakharov type systems", Invent. Math. 172:3 (2008), 535-583. MR 2009f:35298 Zbl 1143.35090

[Merle 1996] F. Merle, "Blow-up results of virial type for Zakharov equations", Comm. Math. Phys. 175:2 (1996), $433-455$. MR 97d:35209 Zbl 0858.35117

[Ozawa and Tsutsumi 1992] T. Ozawa and Y. Tsutsumi, "The nonlinear Schrödinger limit and the initial layer of the Zakharov equations", Differential Integral Equations 5:4 (1992), 721-745. MR 93d:76079 Zbl 0754.35163

[Ozawa and Tsutsumi 1993/94] T. Ozawa and Y. Tsutsumi, "Global existence and asymptotic behavior of solutions for the Zakharov equations in three space dimensions”, Adv. Math. Sci. Appl. 3 (1993/94), 301-334. MR 95h:35217 Zbl 0811.35004

[Schochet and Weinstein 1986] S. H. Schochet and M. I. Weinstein, "The nonlinear Schrödinger limit of the Zakharov equations governing Langmuir turbulence”, Comm. Math. Phys. 106:4 (1986), 569-580. MR 87j:35227 Zbl 0639.76054

[Shimomura 2004] A. Shimomura, "Scattering theory for Zakharov equations in three-dimensional space with large data", Commun. Contemp. Math. 6:6 (2004), 881-899. MR 2005h:35262 Zbl 1203.35183

[Sulem and Sulem 1999] C. Sulem and P.-L. Sulem, The nonlinear Schrödinger equation: self-focusing and wave collapse, Applied Mathematical Sciences 139, Springer, New York, 1999. MR 2000f:35139 Zbl 0928.35157

[Takaoka 1999] H. Takaoka, "Well-posedness for the Zakharov system with the periodic boundary condition", Differential Integral Equations 12:6 (1999), 789-810. MR 2000j:35250 Zbl 1022.35069

[Zakharov 1972] V. E. Zakharov, “Collapse of Langmuir waves”, Zh. Eksp. Teor. Fiz. 62 (1972), 1745-1759. In Russian; translated in Sov. Physics JETP 35:5 (1972), 908-914.

Received 4 Apr 2015. Accepted 3 Sep 2015.

IOAN BEJENARU: ibejenaru@math.ucsd.edu

Department of Mathematics, University of California, San Diego, 9500 Gilman Dr, La Jolla, CA 92093-0112, United States

ZIHUA GUO: zihua.guo@monash.edu

School of Mathematical Sciences, Monash University, Melbourne VIC 3800, Australia

and

LMAM, School of Mathematical Sciences, Peking University, Beijing 100871, China

SEBASTIAN HeRR: herr@math.uni-bielefeld.de

Fakultät für Mathematik, Universität Bielefeld, Postfach 100131, D-33501 Bielefeld, Germany

KENJI NAKANISHI: nakanishi@ist.osaka-u.ac.jp

Department of Pure and Applied Mathematics, Osaka University, Graduate School of Information Science and Technology, Osaka 560-0043, Japan 


\title{
Analysis \& PDE
}

\author{
msp.org/apde
}

\section{EDITORS}

EDITOR-IN-CHIEF

\author{
Maciej Zworski \\ zworski@math.berkeley.edu \\ University of California \\ Berkeley, USA
}

BOARD OF EDITORS

Nicolas Burq Université Paris-Sud 11, France

nicolas.burq@math.u-psud.fr

Sun-Yung Alice Chang Princeton University, USA

chang@math.princeton.edu

Michael Christ University of California, Berkeley, USA

mchrist@math.berkeley.edu

Charles Fefferman Princeton University, USA

cf@math.princeton.edu

Ursula Hamenstaedt Universität Bonn, Germany

ursula@math.uni-bonn.de

Vaughan Jones U.C. Berkeley \& Vanderbilt University vaughan.f.jones@vanderbilt.edu

Herbert Koch Universität Bonn, Germany koch@math.uni-bonn.de

Izabella Laba University of British Columbia, Canada ilaba@math.ubc.ca

Gilles Lebeau Université de Nice Sophia Antipolis, France lebeau@unice.fr

László Lempert Purdue University, USA lempert@math.purdue.edu

Richard B. Melrose Massachussets Institute of Technology, USA rbm@math.mit.edu

Frank Merle Université de Cergy-Pontoise, France Frank.Merle@u-cergy.fr

William Minicozzi II Johns Hopkins University, USA minicozz@math.jhu.edu

Werner Müller Universität Bonn, Germany mueller@math.uni-bonn.de
Yuval Peres

Gilles Pisier

Tristan Rivière

Igor Rodnianski

Wilhelm Schlag

Sylvia Serfaty

Yum-Tong Siu

Terence Tao

Michael E. Taylor

Gunther Uhlmann

András Vasy

Dan Virgil Voiculescu

Steven Zelditch
University of California, Berkeley, USA

peres@stat.berkeley.edu

Texas A\&M University, and Paris 6

pisier@math.tamu.edu

ETH, Switzerland

riviere@math.ethz.ch

Princeton University, USA

irod@math.princeton.edu

University of Chicago, USA

schlag@math.uchicago.edu

New York University, USA

serfaty@cims.nyu.edu

Harvard University, USA siu@math.harvard.edu

University of California, Los Angeles, USA tao@math.ucla.edu

Univ. of North Carolina, Chapel Hill, USA met@math.unc.edu

University of Washington, USA gunther@math.washington.edu

Stanford University, USA andras@math.stanford.edu

University of California, Berkeley, USA

dvv@math.berkeley.edu

Northwestern University, USA

zelditch@math.northwestern.edu

PRODUCTION

production@msp.org

Silvio Levy, Scientific Editor

See inside back cover or msp.org/apde for submission instructions.

The subscription price for 2015 is US \$205/year for the electronic version, and \$390/year ( $\$ 55$, if shipping outside the US) for print and electronic. Subscriptions, requests for back issues from the last three years and changes of subscribers address should be sent to MSP.

Analysis \& PDE (ISSN 1948-206X electronic, 2157-5045 printed) at Mathematical Sciences Publishers, 798 Evans Hall \#3840, c/o University of California, Berkeley, CA 94720-3840, is published continuously online. Periodical rate postage paid at Berkeley, CA 94704, and additional mailing offices.

APDE peer review and production are managed by EditFLOw ${ }^{\circledR}$ from MSP.

\section{PUBLISHED BY}

mathematical sciences publishers

nonprofit scientific publishing

http://msp.org/

(C) 2015 Mathematical Sciences Publishers 


\section{ANALYSIS \& PDE}

\section{Volume $8 \quad$ No. $8 \quad 2015$}

Semilinear wave equations on asymptotically de Sitter, Kerr-de Sitter and Minkowski space- 1807 times

PETER HINTZ and ANDRÁs VASY

A junction condition by specified homogenization and application to traffic lights

1891

Giulio Galise, CYRIL IMberT and RÉGIS MONNEAU

Existence and classification of singular solutions to nonlinear elliptic equations with a gradient 1931 term

JOSHuA CHING and FloriCA CîRSTEA

A topological join construction and the Toda system on compact surfaces of arbitrary genus 1963 AlEKS JEVNIKAR, SADOK KALlEL and ANDREA MALCHIOdI

Well-posedness and scattering for the Zakharov system in four dimensions

2029

IoAn Bejenaru, Zihua Guo, Sebastian Herr and Kenji NaKanishi 